Collection SFN 9 (2008) 65-85

(C) EDP Sciences, Les Ulis

DOI: $10.1051 / \mathrm{sfn}: 2008006$

\title{
Structures magnétiques, diffraction de neutrons et symétrie
}

\author{
F. Bourée ${ }^{1}$ et J. Rodríguez-Carvajal ${ }^{2}$ \\ ${ }^{1}$ LLB [CEA-CNRS], CEA/Saclay, 91191 Gif-sur-Yvette Cedex, France \\ 2 Institut Laue-Langevin, BP. 156, 38042 Grenoble Cedex 9, France
}

\begin{abstract}
Magnetic structure determination (description of the microscopic arrangement of magnetic moments in a crystal) requires neutron diffraction studies. Magnetic neutron powder diffraction is, and will remain in the future, the most straightforward technique to determine magnetic structures as a function of temperature, pressure... We will show here the successive steps of a magnetic structure determination, from the experiment (2-axis diffractometer) to the results: commensurate and/or incommensurate longrange magnetic order, via data analysis. A special attention is devoted to symmetry analysis. Examples are selected in the $\mathrm{R}_{2} \mathrm{~T}_{2} \mathrm{X}$ system (with $\mathrm{R}=$ Lanthanide or Uranium; $\mathrm{T}=$ Transition Metal, $\mathrm{X}=\mathrm{In}$ or $\mathrm{Sn}$ ).
\end{abstract}

\begin{abstract}
Résumé. La diffraction de neutrons est à l'heure actuelle, et restera encore pour un bon nombre d'années, la technique la plus directe de détermination des structures magnétiques, en fonction de la température, de la pression... Les étapes successives d'une telle détermination structurale (structures ferromagnétiques, antiferromagnétiques...) seront présentées dans cet exposé, depuis l'expérience (diffractomètres 2-axes) jusqu'aux résultats (structures magnétiques commensurables et/ou incommensurables), avec une attention particulière réservée à la technique d'analyse de symétrie. Les exemples choisis appartiennent à la «série » $R_{2} T_{2} X(R=$ Lanthanide ou Uranium; $T=$ Métal de Transition; $X=$ In ou $S n)$.
\end{abstract}

\section{INTRODUCTION}

\subsection{Diffraction de neutrons}

La diffraction de neutrons, sur poudre ou monocristal, est l'un des outils privilégiés de l'étude du magnétisme. Nous nous intéresserons dans ce cours à la seule diffraction sur poudre, en montrant à partir d'exemples (simples) les différentes étapes de résolution d'une structure magnétique.

On rend compte de l'interaction neutron-atome par une amplitude de diffusion, A, qui comprend essentiellement trois termes :

$$
\mathrm{A}=\mathrm{b}+2 \mathrm{BI} \cdot \mathbf{S}+\left(\gamma \mathrm{r}_{0} / 2\right) \mathrm{f}(\mathrm{K}) \mathbf{M}_{\perp} \cdot \mathbf{S}
$$

- le premier est d'origine nucléaire : b est isotrope, caractéristique du noyau (il varie avec l'isotope considéré) et conserve pour tous les noyaux le même ordre de grandeur $\left(10^{-12} \mathrm{~cm}\right)$.

- le second, 2BI $\cdot \mathbf{S}$, traduit l'interaction entre le spin du neutron $\mathbf{S}$ et celui du noyau $\mathbf{I}$. Dans le cas usuel d'un échantillon non polarisé $(\langle\mathbf{I}\rangle=0)$, ce terme n'apporte aucune contribution de diffusion cohérente.

- le troisième, $\left(\left(\gamma \mathrm{r}_{0} / 2\right) \mathrm{f}(\mathrm{K}) \mathbf{M}_{\perp} \cdot \mathbf{S}\right.$, traduit l'interaction (dipolaire) entre le spin du neutron $\mathbf{S}$ et le moment magnétique électronique $\mathbf{M}$ de l'atome considéré (contributions de spin et d'orbite). Dans l'expression ci-dessus, $\mathrm{f}(\mathrm{K})$ est le facteur de forme magnétique de l'atome (ion) considéré et $\mathbf{M}_{\perp}$ la composante de moment magnétique perpendiculaire au vecteur de diffusion $\mathbf{K}$, soit :

$$
\mathbf{M}_{\perp}=(\mathbf{K} / \mathrm{K}) \wedge[\mathbf{M} \wedge(\mathbf{K} / \mathrm{K})]=\mathbf{M}-[\mathbf{M} \cdot(\mathbf{K} / \mathrm{K})](\mathbf{K} / \mathrm{K}) .
$$

Notons, fait important, que les amplitudes de diffusion nucléaire, b, et magnétique, $\left(\gamma \mathrm{r}_{0} / 2\right) \mathrm{f}(\mathrm{K}) \mathbf{M}_{\perp} \cdot \mathbf{S}$, qui ont été introduites dans l'expression (1), sont du même ordre de grandeur $\left(10^{-12} \mathrm{~cm}\right)$ : le moment magnétique étant exprimé en magnétons de $\mathrm{Bohr}\left(\mu_{\mathrm{B}}\right)$, le terme $\left(\gamma \mathrm{r}_{0} / 2\right)$ produit de $\gamma$ par $\mathrm{r}_{0}$, rayon classique de l'électron, est alors égal à $0.27 \cdot 10^{-12} \mathrm{~cm}$. 
La diffraction de neutrons (interaction neutron - solide cristallisé; diffusion élastique cohérente) fera ainsi intervenir de manière «parallèle»le facteur de structure nucléaire $F_{N}(\mathbf{K})$ et le «vecteur d'interaction magnétique », $\mathbf{F}_{\perp \mathrm{M}}(\mathbf{K}) \cdot \mathbf{F}_{\perp \mathrm{M}}(\mathbf{K})$ est la composante du facteur de structure magnétique $\mathbf{F}_{\mathrm{M}}(\mathbf{K})$ perpendiculaire au vecteur de diffusion $\mathbf{K}$. Il s'agit d'un vecteur dont les composantes sont des nombres complexes. Pour un faisceau de neutrons incidents non polarisé, l'intensité totale observée sera proportionnelle à la somme des intensités nucléaires et magnétiques, soit :

$$
\left\{\mathrm{F}_{\mathrm{N}}(\mathbf{K})\right\}^{2}+\left\{\left|\mathbf{F}_{\perp \mathrm{M}}(\mathbf{K})\right|\right\}^{2} .
$$

\subsection{Structures magnétiques}

Afin de disposer de tous les éléments nécessaires à la détermination d'une structure magnétique, il nous faut maintenant indiquer de quelle manière nous définissons cette structure. La structure magnétique étant périodique, le moment magnétique d'un atome situé au point $\mathbf{r}_{l, j}=\mathbf{R}_{l}+\mathbf{r}_{j}$ peut être écrit sous la forme :

$$
\mathbf{M}_{l, j}=\sum_{\mathbf{k}} \mathbf{m}_{j}(\mathbf{k}) \exp \left(-2 \mathrm{i} \pi \mathbf{k} \cdot \mathbf{R}_{l}\right)
$$

Dans cette expression $\mathbf{m}_{j}(\mathbf{k})$ est la composante de Fourier, associée au vecteur de propagation $\mathbf{k}$, du moment magnétique correspondant à l'atome situé en position $\mathbf{r}_{j}$ dans la maille primitive. On a bien évidemment $\mathbf{m}_{j}(-\mathbf{k})=\mathbf{m}_{j}(\mathbf{k})^{*}$.

Le calcul de l'intensité magnétique diffractée par l'ensemble du cristal, à partir des formules (1-4), conduit alors, si le résultat est différent de zéro, à la condition $\mathbf{K}=\mathbf{G}+\mathbf{k}$, l'opération $\sum_{\mathbf{R}_{1}}$ faisant apparaître la fonction de Dirac $\delta[\mathbf{K}-(\mathbf{G}+\mathbf{k})]$. Cette condition est l'analogue de la condition $\mathbf{K}=\mathbf{G}$ pour une réflexion purement nucléaire, $\mathbf{G}$ étant dans un cas comme dans l'autre un vecteur du réseau réciproque du cristal.

L'expression du facteur de structure magnétique pour une maille est alors :

$$
\mathbf{F}_{M}(\mathbf{G}+\mathbf{k})=0.27 \cdot 10^{-12} \sum_{j} f_{j}(\mathbf{G}+\mathbf{k}) \mathbf{m}_{j}(\mathbf{k}) \exp \left\{2 \pi i(\mathbf{G}+\mathbf{k}) \mathbf{r}_{j}\right\}
$$

Le vecteur d'interaction magnétique, qui est la composante perpendiculaire au vecteur de diffusion $\mathbf{K}=\mathbf{G}+\mathbf{k}$, de l'expression (1), soit :

$$
\mathbf{F}_{\perp M}(\mathbf{K})=(\mathbf{K} / \mathrm{K}) \wedge\left[\mathbf{F}_{\mathrm{M}}(\mathbf{K}) \wedge(\mathbf{K} / \mathrm{K})\right]=\mathbf{F}_{\mathrm{M}}(\mathbf{K})-\left[\mathbf{F}_{\mathrm{M}}(\mathbf{K}) \cdot(\mathbf{K} / \mathrm{K})\right](\mathbf{K} / \mathrm{K})
$$

devient, en fonction des coefficients de Fourier $\mathbf{m}_{j}(\mathbf{k})$ :

$$
\mathbf{F}_{\perp M}(\mathbf{K})=0.27 \cdot 10^{-12} \sum_{j} f_{j}(\mathbf{K})\left\{\mathbf{m}_{j}(\mathbf{k})-\left(\mathbf{e} \cdot \mathbf{m}_{j}(\mathbf{k})\right) \cdot \mathbf{e}\right\} \exp \left\{2 \pi i \mathbf{K} \mathbf{r}_{j}\right\}
$$

e étant le vecteur unitaire parallèle au vecteur de diffusion $\mathbf{K}$.

Les cas les plus simples de description de structure magnétique en fonction des coefficients de Fourier $\mathbf{m}_{j}(\mathbf{k})$ correspondent aux structures de vecteur de propagation $\mathbf{k}=1 / 2 \mathbf{G}$ (ce qui inclut implicitement le cas $\mathbf{k}=0$ ). Lorsque $\mathbf{k}=1 / 2 \mathbf{G}$, les moments magnétiques sont en effet identiques, au signe près, aux coefficients de Fourier qui sont alors réels : $\mathbf{M}_{l, j}=\mathbf{m}_{j}(\mathbf{k})(-1)^{\mathbf{G . R} l}=\mathbf{m}_{j}(-1)^{n(l)}$. En particulier, l'expression du facteur d'une structure magnétique $\mathbf{k}=0$ est alors :

$$
\mathbf{F}_{\mathrm{M}}(\mathbf{G})=0.27 \cdot 10^{-12} \sum_{j} \mathrm{f}_{j}(\mathbf{G}) \mathbf{m}_{\mathrm{j}} \exp \left\{2 i \pi \mathbf{G} \cdot \mathbf{r}_{j}\right\}
$$

analogue à celle du facteur de structure nucléaire $\mathrm{F}_{\mathrm{N}}(\mathbf{K}=\mathbf{G})$ rappelée ci-dessous :

$$
\mathrm{F}_{\mathrm{N}}(\mathbf{G})=\sum_{j} \mathrm{~b}_{j} \exp \left\{2 i \pi \mathbf{G} \cdot \mathbf{r}_{j}\right\}
$$


Bien entendu, les structures magnétiques observées ne sont pas toujours de ce type. Nombre de structures antiferromagnétiques, commensurables et/ou incommensurables, colinéaires ou non colinéaires, ont ainsi été observées. Nous nous limiterons dans ce document aux structures magnétiques obtenues dans la série $\mathrm{R}_{2} \mathrm{~T}_{2} \mathrm{X}$ [D. Laffargue, PhD, Université de Bordeaux, 1997].

\section{2. $\mathbf{R}_{2} \mathbf{T}_{2} \mathrm{X}$ : STRUCTURES MAGNÉTIQUES COMMENSURABLES ET INCOMMENSURABLES}

\section{$2.1 \mathbf{R}_{2} T_{2} X$ : structure cristalline}

Les composés $\mathrm{R}_{2} \mathrm{~T}_{2} \mathrm{X}$ cristallisent lorsque $\mathrm{T}=\mathrm{Fe}, \mathrm{Co}, \mathrm{Ni}, \mathrm{Ru}, \mathrm{Rh}, \mathrm{Pd}$ et $\mathrm{X}=\mathrm{Sn}$, In dans une structure quadratique de type $\mathrm{U}_{3} \mathrm{Si}_{2}$. Dans le groupe d'espace $\mathrm{P} 4 / \mathrm{mbm}$, l'élément R (Uranium ou Terre Rare) occupe le site $4 \mathrm{~h}\left[\mathrm{x}_{\mathrm{R}} 1 / 2+\mathrm{x}_{\mathrm{R}} 1 / 2\right]$, l'élément $\mathrm{T}$ le site $4 \mathrm{~g}\left[\mathrm{x}_{\mathrm{T}} 1 / 2+\mathrm{x}_{\mathrm{T}} 0\right]$ et l'élément $\mathrm{X}$ le site $2 \mathrm{a}\left[\begin{array}{lll}0 & 0 & 0\end{array}\right]$. Cette structure peut donc être décrite par l'empilement selon l'axe quadratique de plans atomiques alternativement constitués d'atomes $(\mathrm{T}, \mathrm{X})$ et R. Nous ne nous intéresserons désormais qu'aux seuls atomes magnétiquement ordonnés de la série $R_{2} T_{2} X$, les atomes $R$.

\section{$2.2 \mathbf{R}_{2} \mathrm{~T}_{2} \mathrm{X}$ : structures magnétiques

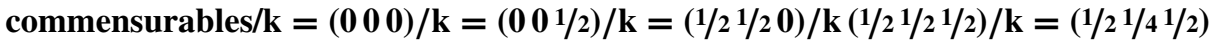

Nous avons choisi d'étudier ces intermétalliques de stoechiométrie 2:2:1 en raison de la très grande variété des structures magnétiques adoptées pour une même structure cristalline (groupe d'espace quadratique $\mathrm{P} 4 / \mathrm{mbm}$ ), selon le choix des éléments $\mathrm{R}$ et $\mathrm{T}$ : ferromagnétisme et/ou antiferromagnétisme, de vecteur(s) de propagation commensurables et/ou incommensurables. Quelques exemples de structures antiferromagnétiques commensurables sont ainsi donnés sur la figure 1, associées respectivement aux vecteurs de propagation $\mathbf{k}=\left(\begin{array}{lll}0 & 0 & 0\end{array}\right)$ [maille magnétique et maille cristalline sont identiques] et $\mathbf{k}=\left(\begin{array}{lll}0 & 0 & 1\end{array} / 2\right)$ [maille magnétique de dimensions $\mathrm{a}, \mathrm{a}, 2 \mathrm{c}$ ]. Les vecteurs commensurables $\mathbf{k}=(1 / 21 / 20), \mathbf{k}=(1 / 21 / 21 / 2)$ et $\mathbf{k}=(1 / 41 / 41 / 2)$ ont été également observés pour d'autres composés de cette famille, dont les structures magnétiques n'ont pas été représentées sur la figure 1.

\section{3 $\mathrm{Ce}_{2} \mathrm{Pd}_{2} \mathrm{Sn}$ : structure magnétique incommensurable}

Les figures 2-6 résument les propriétés magnétiques de $\mathrm{Ce}_{2} \mathrm{Pd}_{2} \mathrm{Sn}$ [9]. La mesure de la susceptibilité magnétique $\chi(\mathrm{T})$ de $\mathrm{Ce}_{2} \mathrm{Pd}_{2} \mathrm{Sn}$ (cf figure 2) montre l'existence pour ce composé de deux transitions magnétiques, à $\mathrm{T}_{\mathrm{N}}=4.8(2) \mathrm{K}$ (transition antiferromagnétique) et $\mathrm{T}_{\mathrm{C}}=3.0(2) \mathrm{K}$ (transition ferromagnétique). Sur la figure 3 sont représentés les diagrammes de diffraction de neutrons caractéristiques de chacun des domaines de température ainsi mis en évidence (domaine paramagnétique (a), antiferromagnétique (b) et ferromagnétique (c)).

Au-dessous de $\mathrm{T}=2.6 \mathrm{~K}$, la structure magnétique obtenue est très simple : les moments de cérium sont identiques et parallèles à l'axe quadratique. De $\mathrm{T}=3.4 \mathrm{~K}$ à $\mathrm{T}=4.75 \mathrm{~K}\left(\mathrm{~T}_{\mathrm{N}}\right)$, le diagramme de diffraction (figure 4) correspond à une structure où les moments magnétiques du cérium restent toujours parallèles à $\mathbf{c}$, mais où leur amplitude varie de manière sinusoïdale en fonction de leur position $\mathbf{r}$ selon la loi $\mathrm{M}(\mathbf{r})=2 \mathrm{~m}(\mathbf{k}) \times \cos (2 \pi \mathbf{k} \cdot \mathbf{r})$, avec $\mathbf{k}=\left(\mathrm{k}_{\mathrm{x}} 00\right)$.

Notons que le vecteur de propagation $\mathbf{k}=\left(\mathrm{k}_{\mathrm{x}} 00\right)$ évolue en fonction de $\mathrm{T}$ (voir figure 5 ) de manière « classique», incommensurable à « haute » température, commensurable à basse température (valeur de lock-in : $\mathbf{k}=\left(\begin{array}{lll}0 & 0 & 0\end{array}\right)$. 


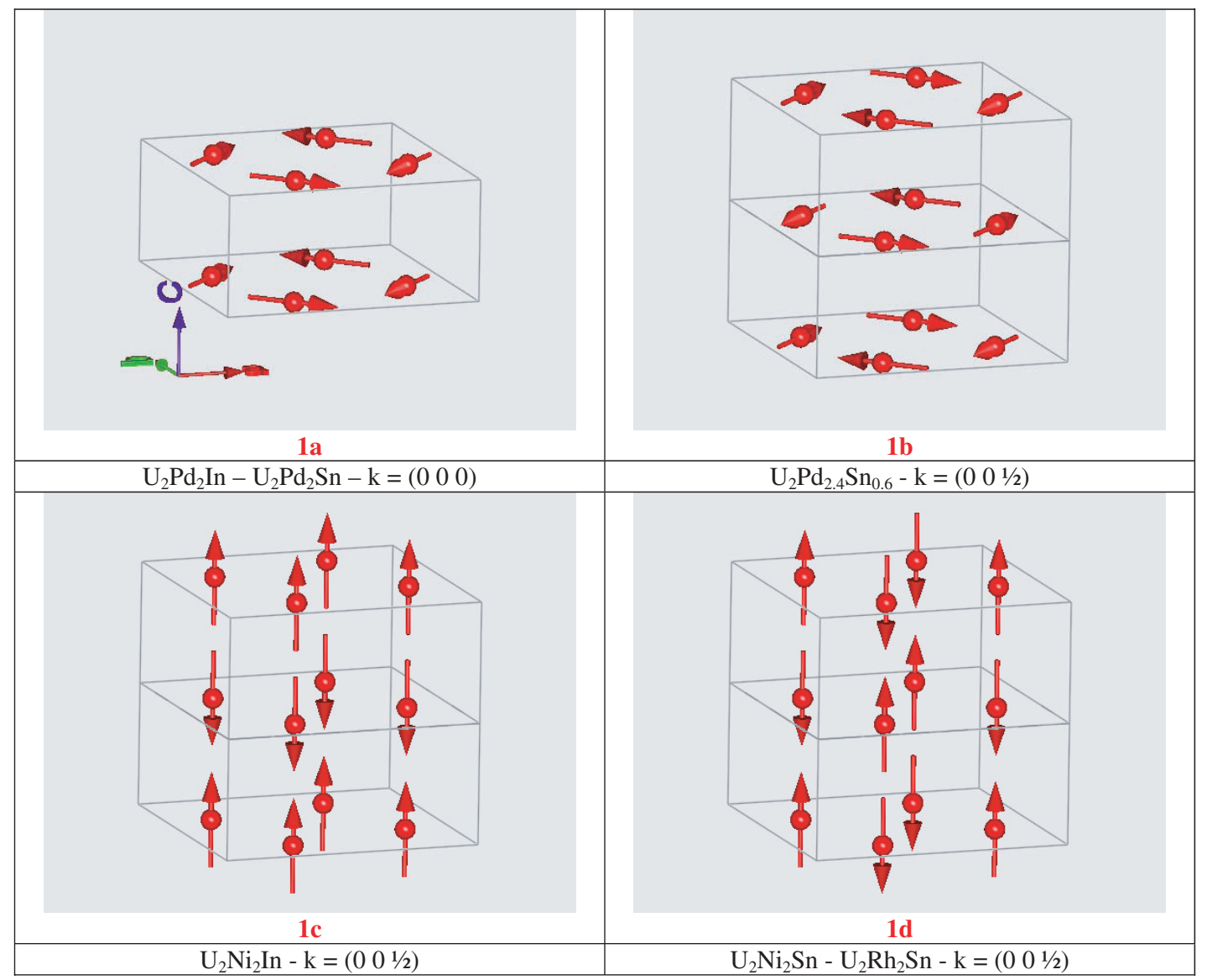

Figure 1. Structures magnétiques commensurables de composés $2: 2: 1$ « $\mathrm{P} 4 / \mathrm{mbm} »[1-8]$.

\section{3. $\mathbf{R}_{2} \mathbf{T}_{2} \mathrm{X}$ : STRUCTURES MAGNÉTIQUES ET ANALYSE DE SYMÉTRIE}

La théorie des groupes est un outil qui peut (et doit ?) être utilisé avant toute détermination de structure magnétique. Nous présentons ci-dessous deux exemples de son utilisation, dans le cas «2:2:1» défini ci-dessus, pour deux vecteurs de propagation, que nous considérerons successivement : l'un commensurable $\mathbf{k}=\left(\begin{array}{lll}0 & 0 & 0\end{array}\right)$, l'autre non $\mathbf{k}=\left(\mathrm{k}_{\mathrm{x}} 00\right)$. Nous supposons donc acquise la connaissance de cette théorie [10-13].

Première étape : définir les conditions du problème, à savoir : quelles sont, dans le groupe d'espace du cristal étudié $(\mathrm{P} 4 / \mathrm{mbm})$, les positions des atomes porteurs de moment magnétique (position de Wyckoff [4h]) et, à partir de l'indexation des pics de Bragg magnétiques, quels sont les vecteurs de propagation permettant de décrire la structure magnétique à déterminer.

L'espace dans lequel sera définie cette structure magnétique est celui des composantes magnétiques des atomes reliés par les éléments de symétrie du groupe du vecteur $\mathbf{k}: \mathrm{G}_{\mathrm{k}}$. Il s'agit d'un sous-groupe de $\mathrm{G}$, qui conserve le vecteur $\mathbf{k}$, à une translation près du réseau réciproque bien entendu. Le cas le plus simple, et nous le traiterons en premier, est celui de $\mathbf{k}=\left(\begin{array}{lll}0 & 0 & 0\end{array}\right)$, où les groupes $\mathrm{G}(\mathrm{P} 4 / \mathrm{mbm})$ et $\mathrm{G}_{\mathrm{k}}$ sont identiques. 


\section{1 $\mathrm{U}_{2} \mathrm{Pd}_{2} \mathrm{In}$ : structure magnétique $\mathrm{k}=\left(\begin{array}{lll}0 & 0 & 0\end{array}\right)$}

Le tableau ci-dessous (tableau I) donne la liste des éléments de symétrie du groupe d'espace P4/mbm

Tableau 1.

\begin{tabular}{|c|c|c|c|c|c|}
\hline Notation & $\begin{array}{c}\text { Loi de } \\
\text { transformation } \\
\text { (vecteur polaire) }\end{array}$ & $\begin{array}{l}\text { Symbole } \\
\text { (symétrie } \\
\text { ponctuelle) }\end{array}$ & Translation & Situation & $\begin{array}{c}\text { Notation } \\
\text { Kovalev } \\
{[13]}\end{array}$ \\
\hline SYM( 1) & $\mathrm{x}, \mathrm{y}, \mathrm{z}$ & 1 & & & $\mathrm{~h}_{1}$ \\
\hline $\operatorname{SYM}(2)$ & $-\mathrm{x},-\mathrm{y}, \mathrm{z}$ & $2 z$ & & & $\mathrm{~h}_{4}$ \\
\hline SYM( 3) & $1 / 2-x, 1 / 2+y,-z$ & $2 y$ & {$\left[\begin{array}{lll}0 & 1 / 2 & 0\end{array}\right]$} & {$\left[\begin{array}{lll}1 / 4 & 0 & 0\end{array}\right]$} & $\mathrm{h}_{3}$ \\
\hline SYM( 4) & $1 / 2+x, 1 / 2-y,-z$ & $2 x$ & {$\left[\begin{array}{lll}1 / 2 & 0 & 0\end{array}\right]$} & {$\left[\begin{array}{lll}0 & 1 / 4 & 0\end{array}\right]$} & $\mathrm{h}_{2}$ \\
\hline SYM( 5) & $1 / 2+y, 1 / 2+x,-z$ & $2_{[110]}$ & {$\left[\begin{array}{lll}1 / 2 & 1 / 2 & 0\end{array}\right]$} & & $\mathrm{h}_{16}$ \\
\hline SYM( 6) & $1 / 2-y, 1 / 2-x,-z$ & $2_{[1 \overline{1} 0]}$ & & {$\left[\begin{array}{lll}0 & 1 / 2 & 0\end{array}\right]$} & $\mathrm{h}_{13}$ \\
\hline SYM( 7) & $\mathrm{y},-\mathrm{x}, \mathrm{z}$ & $4_{z}^{3}$ & & & $\mathrm{~h}_{15}$ \\
\hline SYM( 8) & $-y, x, z$ & $4 z$ & & & $\mathrm{~h}_{14}$ \\
\hline $\operatorname{SYM}(9)$ & $-\mathrm{x},-\mathrm{y},-\mathrm{z}$ & $\overline{1}$ & & & $\mathrm{~h}_{25}$ \\
\hline $\operatorname{SYM}(10)$ & $\mathrm{x}, \mathrm{y},-\mathrm{z}$ & $m_{z}$ & & & $\mathrm{~h}_{28}$ \\
\hline SYM (11) & $1 / 2+x, 1 / 2-y, z$ & $m_{y}$ & {$\left[\begin{array}{lll}1 / 2 & 0 & 0\end{array}\right]$} & {$\left[\begin{array}{lll}0 & 1 / 4 & 0\end{array}\right]$} & $\mathrm{h}_{27}$ \\
\hline $\operatorname{SYM}(12)$ & $1 / 2-x, 1 / 2+y, z$ & $m_{x}$ & {$\left[\begin{array}{lll}0 & 1 / 2 & 0\end{array}\right] \mathrm{b}$} & {$\left[\begin{array}{lll}1 / 4 & 0 & 0\end{array}\right]$} & $\mathrm{h}_{26}$ \\
\hline SYM(13) & $1 / 2-y, 1 / 2-x, z$ & $m_{[110]}$ & & {$\left[\begin{array}{lll}0 & 1 / 2 & 0\end{array}\right]$} & $\mathrm{h}_{40}$ \\
\hline $\operatorname{SYM}(14)$ & $1 / 2+y, 1 / 2+x, z$ & $m_{[1 \overline{1} 0]}$ & {$\left[\begin{array}{lll}1 / 2 & 1 / 2 & 0\end{array}\right]$} & & $\mathrm{h}_{37}$ \\
\hline $\operatorname{SYM}(15)$ & $-\mathrm{y}, \mathrm{x},-\mathrm{z}$ & $\overline{4_{z}^{3}}$ & & & $\mathrm{~h}_{39}$ \\
\hline $\operatorname{SYM}(16)$ & $\mathrm{y},-\mathrm{x},-\mathrm{z}$ & $\overline{4}$ & & & $\mathrm{~h}_{38}$ \\
\hline
\end{tabular}

Les atomes «magnétiques » de $\mathrm{U}_{2} \mathrm{Pd}_{2} \mathrm{In}$, liés par la symétrie du groupe $\mathrm{G}_{\mathrm{k}}$, sont les atomes d'uranium, qui occupent le site (4h), et de coordonnées respectives :

\begin{tabular}{|c|c|c|c|}
\hline $\mathrm{U}_{1}$ & $\mathrm{x}_{\mathrm{U}}$ & $1 / 2+\mathrm{x}_{\mathrm{U}}$ & $1 / 2$ \\
\hline $\mathrm{U}_{2}$ & $1-\mathrm{X}_{\mathrm{U}}$ & $1 / 2-\mathrm{X}_{\mathrm{U}}$ & $1 / 2$ \\
\hline $\mathrm{U}_{3}$ & $1 / 2-\mathrm{X}_{\mathrm{U}}$ & $\mathrm{X}_{\mathrm{U}}$ & $1 / 2$ \\
\hline $\mathrm{U}_{4}$ & $1 / 2+\mathrm{X}_{\mathrm{U}}$ & $1-\mathrm{X}_{\mathrm{U}}$ & $1 / 2$ \\
\hline
\end{tabular}

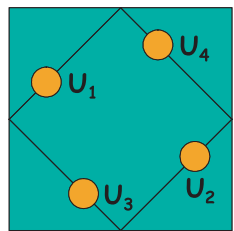

et de moments magnétiques respectifs $\mathbf{M}_{1}, \mathbf{M}_{2}, \mathbf{M}_{3}$ et $\mathbf{M}_{4}$.

Dans l'espace des moments magnétiques $\mathbf{M}_{1-4}$, espace de dimension $4 \times 3=12$ (4 atomes, 3 composantes pour chaque moment magnétique), chacun des éléments de symétrie du groupe $\mathrm{G}_{\mathrm{k}}$ est représenté par une matrice carrée $12 \times 12$, qui traduit les lois de transformations des moments $\mathbf{M}_{1}, \mathbf{M}_{2}$, $\mathbf{M}_{3}$ et $\mathbf{M}_{4}$, tant du point de vue position dans l'espace (permutation des atomes $\mathrm{U}_{1-4}$ ), qu'orientation des moments magnétiques. Rappelons qu'un moment magnétique est un vecteur axial, et que pour un tel vecteur les lois de transformation sont différentes de celles d'un vecteur polaire. La figure ci-dessous (Figure 6) rappelle ainsi l'action d'un miroir (m) sur les composantes d'un vecteur axial : la composante parallèle au miroir est conservée, la composante perpendiculaire est transformée en son opposée. 


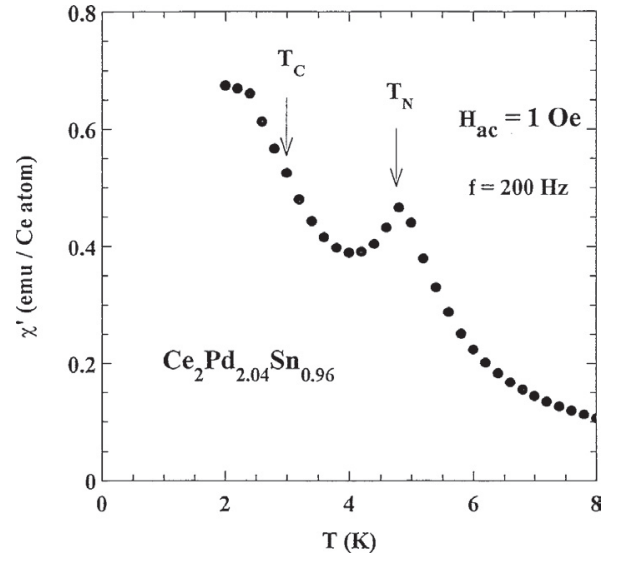

Figure 2. $\mathrm{Ce}_{2} \mathrm{Pd}_{2} \mathrm{Sn}$, susceptibilité magnétique.

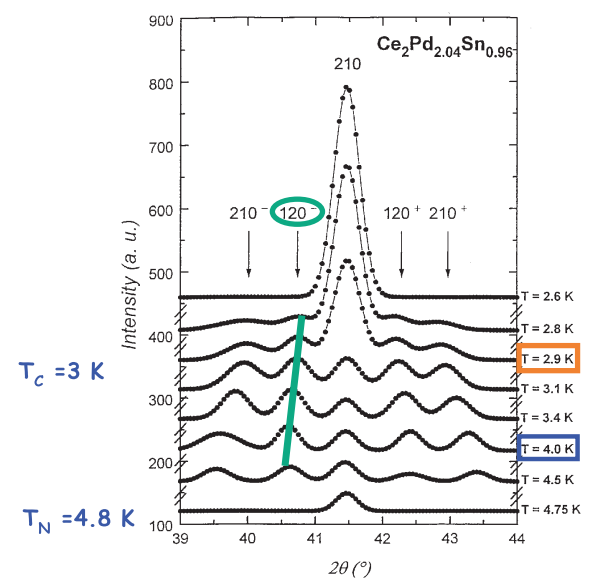

Figure 4. Evolution thermique du diagramme de diffraction de neutrons de $\mathrm{Ce}_{2} \mathrm{Pd}_{2} \mathrm{Sn}$ au voisinage de la raie nucléaire (210). L'indexation des différents satellites observés $(210)^{-},(120)^{-},(120)^{+}$et $(210)^{+}$ est associée au vecteur de propagation $\mathbf{k}=\left(\mathbf{k}_{\mathrm{x}} 00\right)$.

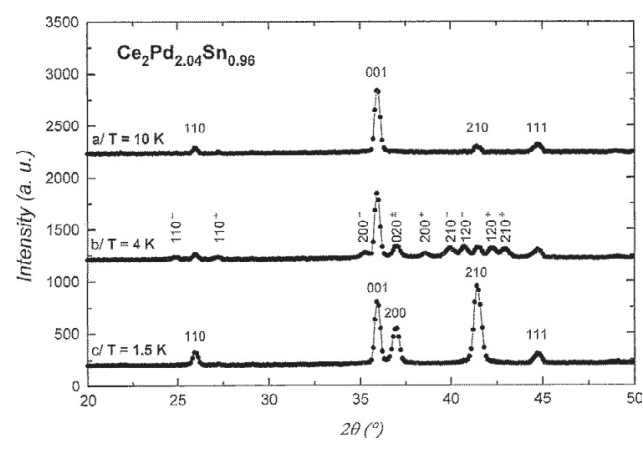

Figure 3. $\mathrm{Ce}_{2} \mathrm{Pd}_{2} \mathrm{Sn}$, diagrammes de diffraction de neutrons (G4.1 - $\lambda=2.425 \AA)$, à $\mathrm{T}=1.5 \mathrm{~K}\left(\mathrm{~T}<\mathrm{T}_{\mathrm{C}}\right), \mathrm{T}=$ $4 \mathrm{~K}\left(\mathrm{~T}_{\mathrm{C}}<\mathrm{T}<\mathrm{T}_{\mathrm{N}}\right)$ et $\mathrm{T}=10 \mathrm{~K}\left(\mathrm{~T}>\mathrm{T}_{\mathrm{N}}\right)$. Les températures de transition $T_{C}$ et $T_{N}$ sont définies sur la figure 2 .

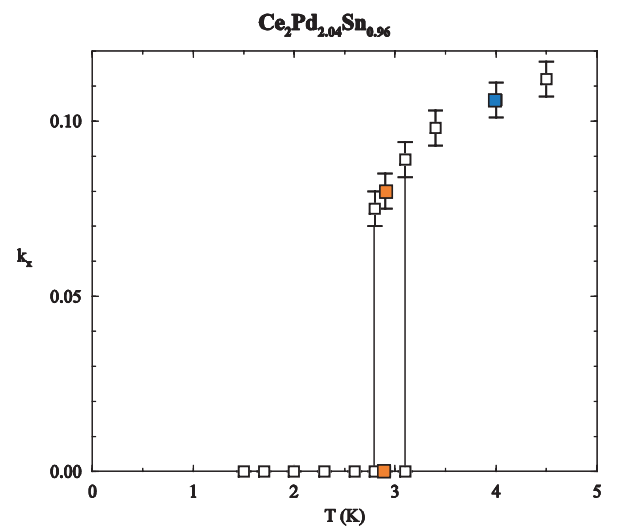

Figure 5. $\mathrm{Ce}_{2} \mathrm{Pd}_{2} \mathrm{Sn}$, vecteur de propagation $\left(\begin{array}{lll}\mathrm{k}_{\mathrm{x}} & 0 & 0\end{array}\right)$ : $\mathrm{k}_{\mathrm{x}}$ vs $\mathrm{T}$. Les deux traits verticaux délimitent la zone de coexistence des vecteurs de propagation commensurable $\left(\mathrm{k}_{\mathrm{x}}=0\right)$ et incommensurable $\left(\mathrm{k}_{\mathrm{x}} \neq 0\right)$.
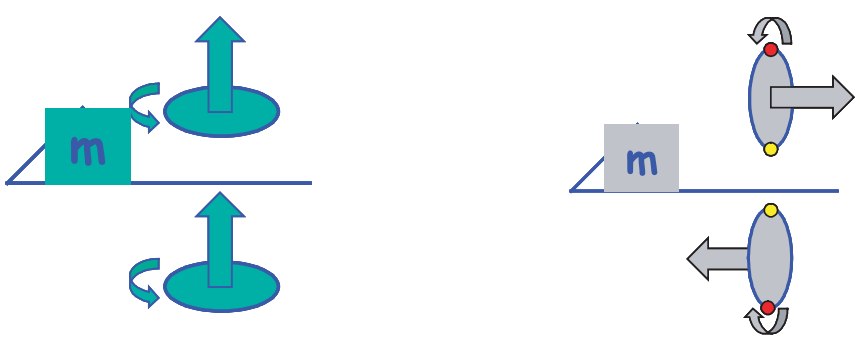

Figure 6. Vecteur axial et symétrie «miroir » : lois de transformation de la "boucle de courant" associée au vecteur axial considéré. 
Dans cet espace de dimension 12, la «matrice magnétique »ainsi définie et associée à l'élément SYM(1), est bien évidemment la matrice identité. L'élément SYM(10), miroir perpendiculaire à l'axe quadratique et passant par l'origine des coordonnées, conserve chacun des atomes $\mathrm{U}_{1-4}$ (à la translation c près). La «matrice magnétique» associée à SYM(10) est alors immédiatement déduite de la figure précédente :

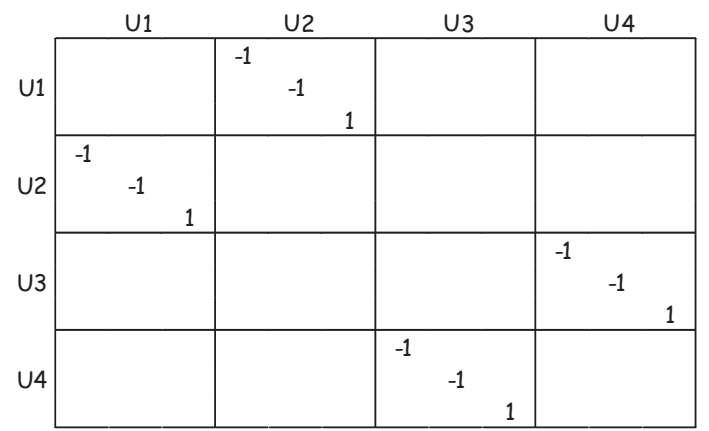

L'ensemble de ces matrices magnétiques constitue une représentation de dimension 12 du groupe $\mathrm{G}_{\mathrm{k}}$, représentation que l'on notera $\Gamma$. Cette représentation «magnétique » est en général réductible, et les règles mathématiques de la théorie des groupes finis permettent alors de la décomposer en Représentations Irréductibles. Le tableau suivant donne la liste des Représentations Irréductibles (RI) du groupe $\mathrm{G}_{\mathrm{k}=(000)}$ considéré.

Tableau 2.

\begin{tabular}{|c|c|c|c|c|c|c|c|c|c|c|c|c|c|c|c|c|}
\hline $\mathrm{P} 4 / \mathrm{mbm}$ & 1 & 2 & 3 & 4 & 5 & 6 & 7 & 8 & 9 & 10 & 11 & 12 & 13 & 14 & 15 & 16 \\
\hline$\Gamma_{1}$ & 1 & 1 & 1 & 1 & 1 & 1 & 1 & 1 & 1 & 1 & 1 & 1 & 1 & 1 & 1 & 1 \\
\hline$\Gamma_{2}$ & 1 & 1 & 1 & 1 & 1 & 1 & 1 & 1 & -1 & -1 & -1 & -1 & -1 & -1 & -1 & -1 \\
\hline$\Gamma_{3}$ & 1 & 1 & 1 & 1 & -1 & -1 & -1 & -1 & 1 & 1 & 1 & 1 & -1 & -1 & -1 & -1 \\
\hline$\Gamma_{4}$ & 1 & 1 & 1 & 1 & -1 & -1 & -1 & -1 & -1 & -1 & -1 & -1 & 1 & 1 & 1 & 1 \\
\hline$\Gamma_{5}$ & 1 & 1 & -1 & -1 & 1 & 1 & -1 & -1 & 1 & 1 & -1 & -1 & 1 & 1 & -1 & -1 \\
\hline$\Gamma_{6}$ & 1 & 1 & -1 & -1 & 1 & 1 & -1 & -1 & -1 & -1 & 1 & 1 & -1 & -1 & 1 & 1 \\
\hline$\Gamma_{7}$ & 1 & 1 & -1 & -1 & -1 & -1 & 1 & 1 & 1 & 1 & -1 & -1 & -1 & -1 & 1 & 1 \\
\hline$\Gamma_{8}$ & 1 & 1 & -1 & -1 & -1 & -1 & 1 & 1 & -1 & -1 & 1 & 1 & 1 & 1 & -1 & -1 \\
\hline$\Gamma_{9}$ & $\begin{array}{ll}1 & 0 \\
0 & 1\end{array}$ & $\begin{array}{rr}-1 & 0 \\
0 & -1\end{array}$ & $\begin{array}{rr}1 & 0 \\
0 & -1\end{array}$ & $\begin{array}{rl}-1 & 0 \\
0 & 1\end{array}$ & $\begin{array}{ll}0 & 1 \\
1 & 0\end{array}$ & $\begin{array}{rr}0 & -1 \\
-1 & 0\end{array}$ & $\begin{array}{cc} & -1 \\
1 & 0\end{array}$ & $\begin{array}{rr}0 & 1 \\
-1 & 0\end{array}$ & $\begin{array}{cc}-1 & 0 \\
0 & -1\end{array}$ & $\begin{array}{ll}1 & 0 \\
0 & 1\end{array}$ & $\begin{array}{rl}-1 & 0 \\
0 & 1\end{array}$ & $\begin{array}{rr}1 & 0 \\
0 & -1\end{array}$ & $\begin{array}{rr}0 & -1 \\
-1 & 0\end{array}$ & $\begin{array}{ll} & 1 \\
1 & 0\end{array}$ & $\begin{array}{rl}0 & 1 \\
-1 & 0\end{array}$ & $\begin{array}{rr}0 & -1 \\
1 & 0\end{array}$ \\
\hline$\Gamma_{10}$ & $\begin{array}{ll}1 & 0 \\
0 & 1 \\
\end{array}$ & $\begin{array}{rr}-1 & 0 \\
0 & -1 \\
\end{array}$ & $\begin{array}{rr}1 & 0 \\
0 & -1 \\
\end{array}$ & $\begin{array}{rr}-1 & 0 \\
0 & 1 \\
\end{array}$ & $\begin{array}{ll}0 & 1 \\
1 & 0 \\
\end{array}$ & $\begin{array}{rr}0 & -1 \\
-1 & 0 \\
\end{array}$ & $\begin{array}{lr}0 & -1 \\
1 & 0 \\
\end{array}$ & $\begin{array}{rr}0 & 1 \\
-1 & 0 \\
\end{array}$ & $\begin{array}{ll}1 & 0 \\
0 & 1 \\
\end{array}$ & $\begin{array}{rr}-1 & 0 \\
0 & -1 \\
\end{array}$ & $\begin{array}{rr}1 & 0 \\
0 & -1 \\
\end{array}$ & $\begin{array}{rr}-1 & 0 \\
0 & 1 \\
\end{array}$ & $\begin{array}{ll}0 & 1 \\
1 & 0 \\
\end{array}$ & $\begin{array}{rr}0 & -1 \\
-1 & 0 \\
\end{array}$ & $\begin{array}{rr}0 & -1 \\
1 & 0 \\
\end{array}$ & $\begin{array}{rr}01 \\
-10 \\
\end{array}$ \\
\hline
\end{tabular}

Huit de ces RI sont de dimension $1\left(\Gamma_{1-8}\right)$ et deux de dimension $2\left(\Gamma_{9-10}\right)$. Les règles d'orthogonalité permettent alors d'obtenir la décomposition suivante : $\Gamma=\Gamma_{2} \oplus \Gamma_{3} \oplus \Gamma_{4} \oplus \Gamma_{6} \oplus \Gamma_{7} \oplus \Gamma_{8} \oplus \Gamma_{9}$ $\oplus 2 \Gamma_{10}$, où l'on se convaincra aisément que la dimension 12 de $\Gamma$ est conservée dans la décomposition obtenue. Les structures magnétiques associées aux RI de dimension 1, pour lesquelles un seul paramètre, la grandeur du moment magnétique, est «disponible», sont représentées sur la figure ci-dessous (Figure 7).

Sur cette figure, les notations $P 4 / m^{\prime} b^{\prime} m^{\prime}, P 4 / m^{\prime} b m^{\prime}$... sont les «groupes de Shubnikov» [14], ou «groupes de couleur», associés aux représentations irréductibles de dimension 1 considérées $\left(\Gamma_{2}, \Gamma_{8}\right.$ ...). Les éléments de symétrie $4, m, b$ et $m$, sont, dans l'ordre, les éléments $\operatorname{SYM}(8), \operatorname{SYM}(10)$, $\operatorname{SYM}(12)$ et $\operatorname{SYM}(13)$ du Tableau I. Pour l'opération de symétrie «primée » \{ symbole «' »\}, le moment magnétique final est le moment magnétique résultat de l'opération de symétrie non « primée », transformé en son opposé. A titre d'illustration, considérons le miroir $m\{\mathrm{SYM}(10)\}$ perpendiculaire à l'axe quadratique 4 . Les atomes $\mathrm{U}_{1-4}$ étant situés sur ce miroir, il devient évident, considérant les lois de transformation des composantes d'un vecteur axial par symétrie « miroir » représentées sur la figure 6, que les moments magnétiques $\mathbf{M}_{1-4}$ qui leur sont associés seront soit perpendiculaires (opération $m$ ), soit parallèles à ce miroir (opération $m^{\prime}$ ). Ce qui est effectivement observé sur la figure 7 . Le lecteur 


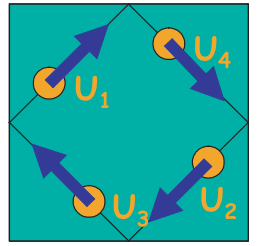

$\Gamma_{2} P 4 / m^{\prime} b^{\prime} m^{\prime}$

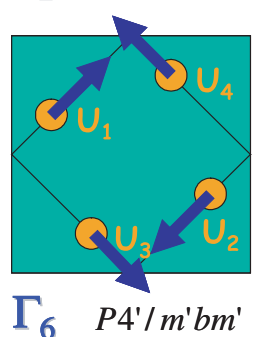

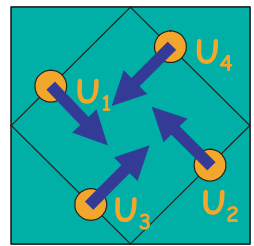

$\Gamma_{8} P 4 / m^{\prime} b m$

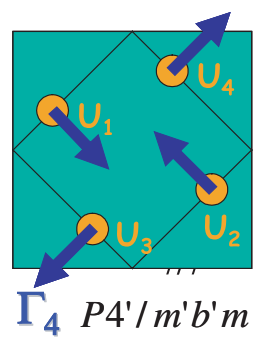

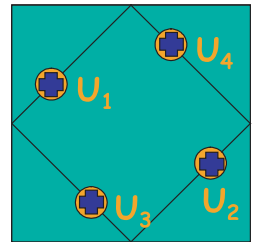

$\Gamma_{7} P 4 / m b^{\prime} m^{\prime}$

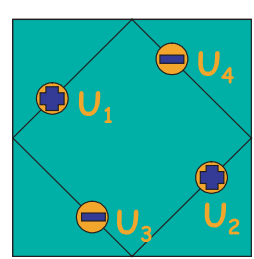

$\Gamma_{3} P 4^{\prime} / \mathrm{mbm}^{\prime}$

Figure 7. Groupe d'espace $\mathrm{P} 4 / \mathrm{mbm}$, vecteur de propagation $\mathbf{k}=(000)$, site $(4 \mathrm{~h})$ : structures magnétiques associées aux RI (Représentations Irréductibles) de dimension $1\left(\Gamma_{2,3,4,6,7,8}\right)$.

effectuera sans difficulté le même travail d'interprétation « géométrique » pour les opérations $4, b$ et $m$, respectivement $\operatorname{SYM}(8), \operatorname{SYM}(12)$ et $\operatorname{SYM}(13)$.

Pour la RI de dimension 2, $\Gamma_{9}$, qui n'intervient qu'une fois dans la décomposition de $\Gamma$ en RI, les moments magnétiques qui définissent la structure sont parallèles à l'axe quadratique, et tels que $\mathbf{M}_{1}+\mathbf{M}_{2}=0$ et $\mathbf{M}_{3}+\mathbf{M}_{4}=0$. Deux paramètres suffisent ainsi à définir cette structure : $\mathbf{M}_{1}$ et $\mathbf{M}_{3}$.
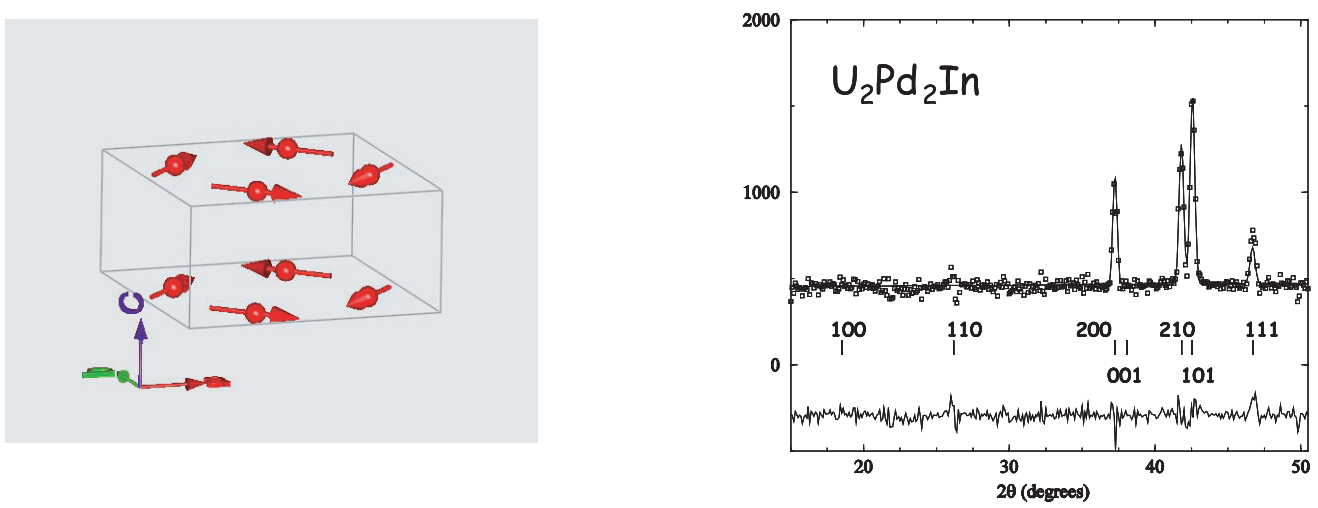

Figure 8. $\mathrm{U}_{2} \mathrm{Pd}_{2} \mathrm{In}$ : structure magnétique $\left[\mathrm{T}<\mathrm{T}_{\mathrm{N}}\right]$ et diagramme de diffraction de neutrons « $[\mathrm{T}=1.5 \mathrm{~K}]-$ $\left[\mathrm{T}>\mathrm{T}_{\mathrm{N}}\right]$ » $(\mathrm{G} 4.1-\lambda=2.425 \AA$ A). Seule la partie «magnétique »de ce spectre est donc représentée à $\mathrm{T}=1.5 \mathrm{~K}$ sur cette figure : points expérimentaux (symboles), spectre calculé (ligne continue), positions des pics de Bragg $2 \theta_{h k l}$ et comparaison expérience-modèle (ligne continue inférieure).

Enfin, pour la RI de dimension $2, \Gamma_{10}$, qui intervient deux fois dans la décomposition de $\Gamma$ en RI, les moments magnétiques qui définissent la structure sont perpendiculaires à l'axe quadratique, et tels que $\mathbf{M}_{1}=\mathbf{M}_{2}$ et $\mathbf{M}_{3}=\mathbf{M}_{4}$. On le voit, quatre paramètres sont alors nécessaires à la description complète de cette structure.

La figure 8 donne la structure magnétique déterminée par diffraction de neutrons pour le composé $\mathrm{U}_{2} \mathrm{Pd}_{2} \mathrm{In}$, ainsi que les résultats de l'affinement de profil (FULLPROF) du diagramme « magnétique » de 
diffraction de neutrons sur poudre à $\mathrm{T}=1.4 \mathrm{~K}$ (diagramme différence $\mathrm{T}=1.4 \mathrm{~K}-\mathrm{T}>\mathrm{T}_{\mathrm{N}}$, données enregistrées sur le diffractomètre 2-axes G4.1 du LLB). La structure de $\mathrm{U}_{2} \mathrm{Pd}_{2} \mathrm{In}$ est celle qui est associée à la représentation irréductible $\Gamma_{6}$; l'affinement du diagramme a conduit à une valeur de moment magnétique $\mathrm{M}_{\mathrm{U}}=1.55 \mu_{\mathrm{B}}$ à $\mathrm{T}=1.4 \mathrm{~K}[4]$.

\section{2 $\mathrm{Ce}_{2} \mathrm{Pd}_{2} \mathrm{Sn}:$ structure magnétique $\mathrm{k}=\left(\mathrm{k}_{\mathrm{x}} \mathbf{0} 0\right)$}

Pour le vecteur de propagation $\mathbf{k}=\mathrm{k}_{\mathrm{x}} \mathbf{a}^{*}$, avec $\mathrm{k}_{\mathrm{x}}$ incommensurable, le groupe $\mathrm{G}_{\mathrm{k}}$, qui conserve $\mathbf{k}$, est un sous-groupe de P4/mbm, uniquement constitué des éléments SYM(1), SYM(4), SYM(10) et SYM(11) du tableau 1. Par souci de clarté des notations dans ce paragraphe, nous les rebaptiserons SYMM(1), SYMM(2), SYMM(3) et SYMM(4). Le tableau 3 est alors l'analogue du tableau 1.

Tableau 3.

\begin{tabular}{|c|c|c|c|c|c|}
\hline Notation & $\begin{array}{c}\text { Loi de } \\
\text { transformation } \\
\text { (vecteur polaire) }\end{array}$ & $\begin{array}{c}\text { Symbole } \\
\text { (symétrie } \\
\text { ponctuelle) }\end{array}$ & Translation & Situation & $\begin{array}{c}\text { Notation } \\
\text { Kovalev } \\
{\left[\begin{array}{l}13\end{array}\right]}\end{array}$ \\
\hline SYMM(1) & $\mathrm{x}, \mathrm{y}, \mathrm{z}$ & 1 & & & $\mathrm{~h}_{1}$ \\
\hline $\mathrm{SYMM}(2)$ & $1 / 2+\mathrm{x}, 1 / 2-\mathrm{y},-\mathrm{z}$ & $2_{x}$ & {$\left[\begin{array}{lll}1 / 2 & 0 & 0\end{array}\right]$} & {$\left[\begin{array}{lll}0 & 1 / 4 & 0\end{array}\right]$} & $\mathrm{h}_{2}$ \\
\hline SYMM(3) & $\mathrm{x}, \mathrm{y},-\mathrm{z}$ & $m_{z}$ & & & $\mathrm{~h}_{28}$ \\
\hline SYMM(4) & $1 / 2+\mathrm{x}, 1 / 2-\mathrm{y}, \mathrm{z}$ & $m_{y}$ & {$\left[\begin{array}{lll}1 / 2 & 0 & 0\end{array}\right]$} & {$\left[\begin{array}{lll}0 & 1 / 4 & 0\end{array}\right]$} & $\mathrm{h}_{27}$ \\
\hline
\end{tabular}

Comme pour $\mathrm{U}_{2} \mathrm{Pd}_{2} \mathrm{In}$, les atomes magnétiques de $\mathrm{Ce}_{2} \mathrm{Pd}_{2} \mathrm{Sn}$ sont les atomes qui occupent le site (4h) de la structure cristalline, sauf que dans le cas présent, $\mathbf{k}=\left(\mathrm{k}_{\mathrm{x}} 00\right)$, avec $\mathrm{k}_{\mathrm{x}} \neq 0$ et $\mathrm{k}_{\mathrm{x}} \neq 1 / 2$, le groupe du vecteur $\mathbf{k}$ les sépare en deux « orbites » distinctes : la première de ces orbites est constituée des atomes $\mathrm{Ce}_{1}$ et $\mathrm{Ce}_{4}$, la seconde de $\mathrm{Ce}_{2}$ et $\mathrm{Ce}_{3}$, aucune opération de symétrie du groupe $\mathrm{G}_{\mathrm{k}}$ ne permettant d'obtenir un atome d'une orbite à partir d'un atome de l'autre. Les coordonnées, dans la maille élémentaire passant par l'origine, de ces deux familles d'atomes sont donc respectivement :

\begin{tabular}{|c|c|c|c|}
\hline $\mathrm{Ce}_{1}$ & $\mathrm{X}_{\mathrm{Ce}}$ & $1 / 2+\mathrm{X}_{\mathrm{Ce}}$ & $1 / 2$ \\
\hline $\mathrm{Ce}_{4}$ & $1 / 2+\mathrm{X}_{\mathrm{Ce}}$ & $1-\mathrm{X}_{\mathrm{Ce}}$ & $1 / 2$ \\
\hline
\end{tabular}

pour l'orbite (1) et, pour l'orbite (2) :

\begin{tabular}{|c|c|c|c|}
\hline $\mathrm{Ce}_{3}$ & $1 / 2-\mathrm{x}_{\mathrm{Ce}}$ & $\mathrm{x}_{\mathrm{Ce}}$ & $1 / 2$ \\
\hline $\mathrm{Ce}_{2}$ & $1-x_{\mathrm{Ce}}$ & $1 / 2-\mathrm{x}_{\mathrm{Ce}}$ & $1 / 2$ \\
\hline
\end{tabular}

À l'image du tableau 2, le tableau 4 donne la liste des Représentations Irréductibles (RI) du groupe $\mathrm{G}_{\mathrm{k}}\left[\mathrm{k}=\left(\mathrm{k}_{\mathrm{x}} 00\right)\right]$ considéré. Dans ce tableau intervient un paramètre de valeur complexe, $\alpha=\exp \left\{2 \mathrm{i} \pi\left[1 / 2 \cdot \mathrm{k}_{\mathrm{x}}\right]\right\}$, qui traduit le déphasage lié à la partie translation de l'opération de symétrie considérée.

\section{Tableau 4.}

\begin{tabular}{|c|c|c|c|c|}
\hline $\boldsymbol{G}_{\mathrm{k}}$ & SYMM(1) & SYMM(2) & SYMM(3) & SYMM(4) \\
\hline$\Gamma_{1}$ & 1 & $\alpha$ & 1 & $\alpha$ \\
\hline$\Gamma_{2}$ & 1 & $\alpha$ & -1 & $-\alpha$ \\
\hline$\Gamma_{3}$ & 1 & $-\alpha$ & 1 & $-\alpha$ \\
\hline$\Gamma_{4}$ & 1 & $-\alpha$ & -1 & $\alpha$ \\
\hline
\end{tabular}


Tableau 5

\begin{tabular}{|c|c|c|c|c|}
\hline$\Gamma$ & $C e_{1}$ & $C e_{4}$ & $C e_{3}$ & $C e_{2}$ \\
\hline$\Gamma_{1}$ & {$[00 w]$} & $\alpha *[00-w]$ & {$\left[00 w^{\prime}\right]$} & $\alpha *\left[00-w^{\prime}\right]$ \\
\hline $2 \Gamma_{2}$ & {$[u v 0]$} & $\alpha *[u-v 0]$ & {$\left[u^{\prime} v^{\prime} 0\right]$} & $\alpha *\left[u^{\prime}-v^{\prime} 0\right]$ \\
\hline$\Gamma_{3}$ & {$[00 w]$} & $\alpha *[00 w]$ & {$\left[00 w^{\prime}\right]$} & $\alpha *\left[00 w^{\prime}\right]$ \\
\hline $2 \Gamma_{4}$ & {$[u v 0]$} & $\alpha *[-u v 0]$ & {$\left[u^{\prime} v^{\prime} 0\right]$} & $\alpha *\left[-u^{\prime} v^{\prime} 0\right]$ \\
\hline
\end{tabular}

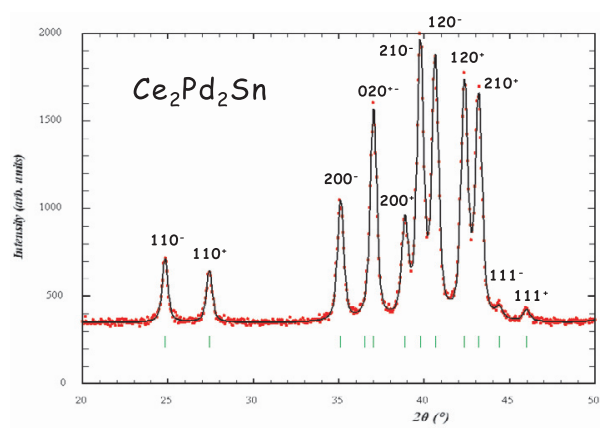

Figure 9. $\mathrm{Ce}_{2} \mathrm{Pd}_{2} \mathrm{Sn}$, diffractogramme calculé $\Gamma_{3}$.

La décomposition de la représentation «magnétique » doit ici s'effectuer séparément pour l'orbite (1) et pour l'orbite (2). Pour chacune d'elles, la représentation magnétique $\Gamma$ est donc de dimension 6; et se décompose en RI selon la relation : Gamma $=\Gamma_{1} \oplus \Gamma_{2} \oplus \Gamma_{3} \oplus 2 \Gamma_{4}$. Les vecteurs de base des RI de cette décomposition sont obtenus par la méthode de l'opérateur de projection et donnés dans le tableau $\mathrm{V}$. Si les résultats sont similaires pour l'ensemble $\left\{\mathrm{Ce}_{1}, \mathrm{Ce}_{4}\right\}$ (orbite (1) et l'ensemble $\left\{\mathrm{Ce}_{3}, \mathrm{Ce}_{2}\right\}$ (orbite (2)), rien au niveau de la théorie des représentations ne permet de lier les paramètres $u, v, w$ et $u^{\prime}, v^{\prime}, w^{\prime}$ associés à une même RI de ce tableau.

Les figures 3 et 9 permettent de comparer les diagrammes de diffraction de neutrons de $\mathrm{Ce}_{2} \mathrm{Pd}_{2} \mathrm{Sn}$ à $\mathrm{T}=4 \mathrm{~K}$ : données expérimentales « G4.1» pour la figure 3, et, pour la figure 9, diffractogramme «magnétique» calculé dans le cas de la Représentation Irréductible Gamma 3 du tableau 5.

La structure magnétique incommensurable de $\mathrm{Ce}_{2} \mathrm{Pd}_{2} \mathrm{Sn}$ est alors représentée, dans le cas où la composante $\mathrm{k}_{\mathrm{x}}$ est égale à 0.105 , sur la figure $10 \mathrm{a}$ pour quatre atomes $\left[\mathrm{Ce}_{1-4}\right.$ ] situés dans une même maille élémentaire, et pour l'ensemble $(10 \mathrm{a} \times \mathrm{a} \times \mathrm{c})$ sur la figure $10 \mathrm{~b}$.

Si l'on rappelle la définition des moments magnétiques donnée dans l'introduction de cet article,

$$
\mathbf{M}_{1, j}=\sum_{k,-k} \mathbf{m}_{\mathrm{j}}(\mathbf{k}) \exp \left(-2 i \pi \mathbf{k} \cdot \mathbf{R}_{\mathrm{l}}\right)
$$

la structure magnétique incommensurable $« \Gamma_{3} » d e \mathrm{Ce}_{2} \mathrm{Pd}_{2} \mathrm{Sn}$ est définie, en utilisant les paramètres introduits dans le tableau $\mathrm{V}$, par les relations suivantes :

$$
\begin{array}{lll}
\text { Orbite (1) } & \mathrm{Ce}_{1} & (00 \mathrm{w}) \exp \left(-2 \mathrm{i} \pi \mathbf{k} \cdot \mathbf{R}_{1}\right)+\left(00 \mathrm{w}^{\star}\right) \exp \left(+2 \mathrm{i} \pi \mathbf{k} \cdot \mathbf{R}_{1}\right) \\
& \mathrm{Ce}_{4} & \left(00 \alpha^{*} \mathbf{w}\right) \exp \left(-2 \mathrm{i} \pi \mathbf{k} \cdot \mathbf{R}_{1}\right)+\left(00 \alpha \mathbf{w}^{\star}\right) \exp \left(+2 \mathrm{i} \pi \mathbf{k} \cdot \mathbf{R}_{1}\right) \\
& \mathrm{Ce}_{3} & \left(00 \mathrm{w}^{\prime}\right) \exp \left(-2 \mathrm{i} \pi \mathbf{k} \cdot \mathbf{R}_{1}\right)+\left(00 \mathrm{w}^{\star \star}\right) \exp \left(+2 \mathrm{i} \pi \mathbf{k} \cdot \mathbf{R}_{1}\right) \\
\text { Orbite(2) } & \mathrm{Ce}_{2} & \left(00 \alpha^{*} \mathbf{w}^{\prime}\right) \exp \left(-2 \mathrm{i} \pi \mathbf{k} \cdot \mathbf{R}_{1}\right)+\left(00 \alpha \mathbf{w}^{\prime \star}\right) \exp \left(+2 \mathrm{i} \pi \mathbf{k} \cdot \mathbf{R}_{1}\right) .
\end{array}
$$

L'affinement du diagramme expérimental permet alors d'obtenir la valeur des paramètres $\mathbf{w}$ et $\mathbf{w}^{\prime}$, soit $\mathbf{w}^{\prime}=\beta * \mathrm{w}$, avec $\beta=\exp \left\{2 \mathrm{i} \pi\left[\left(\mathrm{x}_{\mathrm{Ce} 3}-\mathrm{x}_{\mathrm{Ce} 1}\right) \cdot \mathrm{k}_{\mathrm{x}}\right]\right\}$, conduisant à l'expression simplifiée $\mathbf{M}_{1, \mathrm{j}}=\mathrm{Mcos}$ $\left\{2 \pi \mathbf{k} \cdot\left(\mathbf{R}_{1}+\mathbf{r}_{\mathrm{j}}\right)\right\} \mathbf{e}_{z}$, où $\mathrm{M}=1.75 \mu_{\mathrm{B}}$ à $\mathrm{T}=4 \mathrm{~K}$, avec $\mathbf{e}_{z}$ vecteur unitaire parallèle à l'axe quadratique $\mathbf{c}$. 
a

b
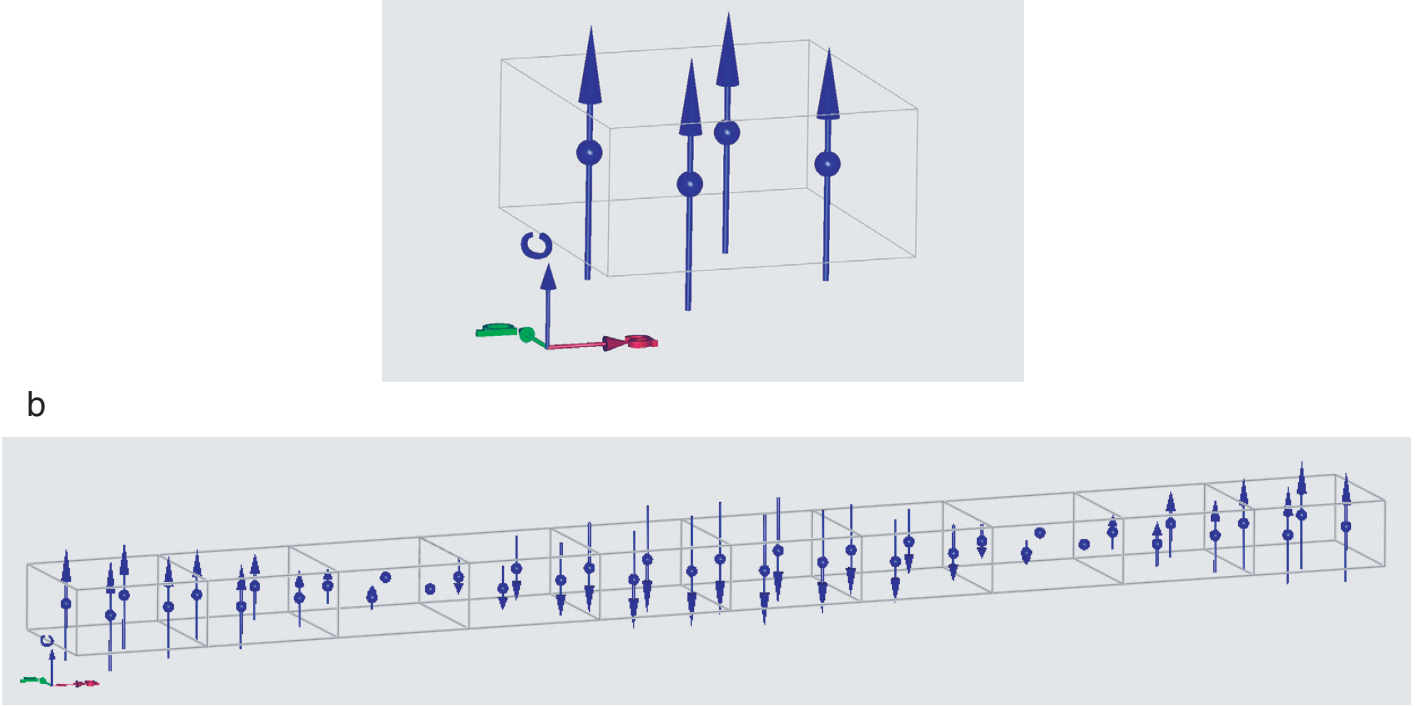

Figure 10. a. $\mathrm{Ce}_{2} \mathrm{Pd}_{2} \mathrm{Sn}$, structure magnétique $\mathrm{T}=4 \mathrm{~K}$. Seule une maille élémentaire est représentée sur cette figure. b. $\mathrm{Ce}_{2} \mathrm{Pd}_{2} \mathrm{Sn}$, structure magnétique sinusoïdale, de vecteur de propagation $\mathrm{k}=\left(\mathrm{k}_{\mathrm{x}}=0.100\right)$.

Bien entendu, les résultats qui ont été présentés en 3.1 (cas « commensurable ») et 3.2 (cas « incommensurable ») sont accessibles via FullProf, et le logiciel dédié aux calculs présentés ci-dessus : BASIREPS. Notons que parmi les fichiers créés par BASIREPS, l'un, d'extension fp, est directement utilisable lors de l'affinement FULLProf de la structure magnétique. A titre indicatif, nous en donnons ci-dessous la version associée au cas incommensurable 2:2:1, $\mathrm{k}_{\mathrm{x}}=0.10 \mathbf{c}^{*}, \Gamma_{3}$ :

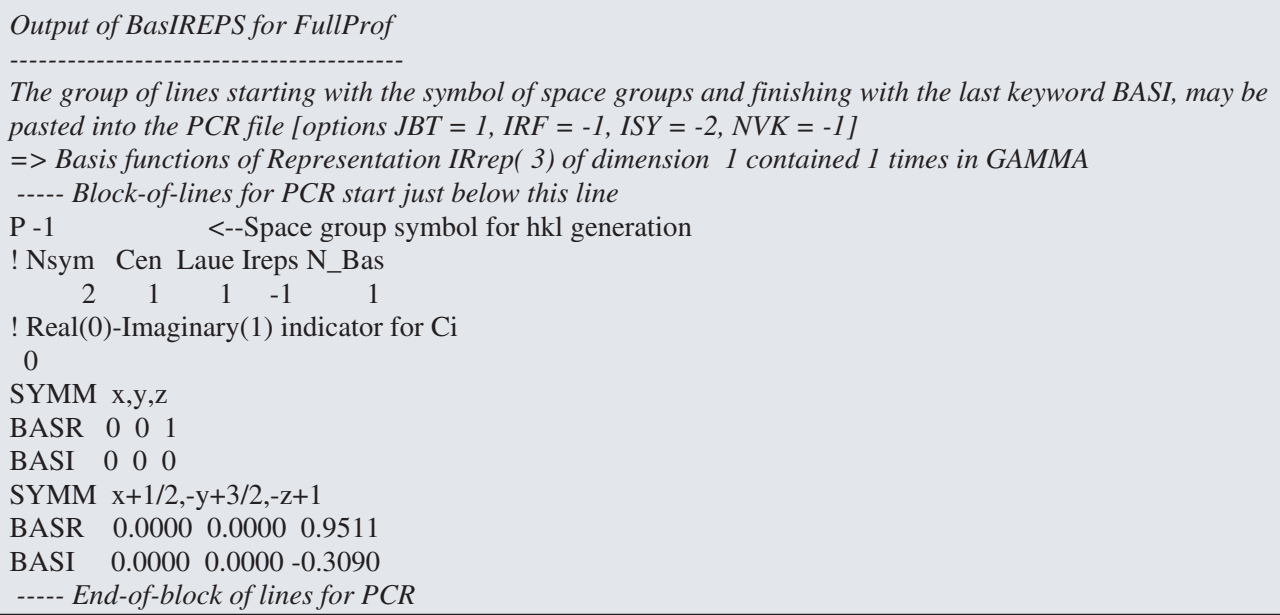

Ce "fichier" concerne ainsi les atomes magnétiques d'une même orbite, $\mathrm{Ce}_{1}[\mathrm{x}$ y z $]$ et $\mathrm{Ce}_{4}[\mathrm{x}+1 / 2$ $\mathrm{y}+3 / 2-\mathrm{z}+1$ ] (orbite (1) par exemple, et les relations entre les composantes BASR, BASI des deux atomes correspondent au déphasage défini par la constante $\alpha^{*}=\exp \left\{-2 \mathrm{i} \pi\left[1 / 2 \cdot \mathrm{k}_{\mathrm{x}}\right]\right\}$ du tableau V. 

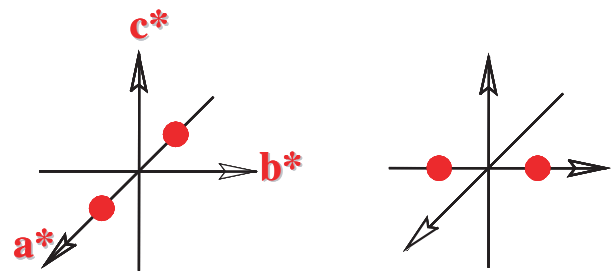

Simple-k \& Domaines Magnétiques
(proportions $\equiv)$

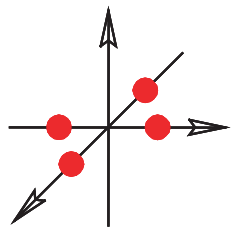

Double-k / Monodomaine

Figure 11. a, b. Structures magnétiques et domaines « $\mathbf{k} »$. En supposant que l'origine des axes $\mathbf{a}^{*}, \mathbf{b}^{*}, \mathbf{c}^{*}$ représente sur ces schémas un nœud $(h k l)$ du réseau réciproque, les schémas ci-dessus indiquent, dans le réseau réciproque du cristal, la position des pics de Bragg que l'on observera pour une structure « simple-k » (Figure 11a / vecteur de propagation $\{\mathbf{k},-\mathbf{k}\}$ ou $\left\{\mathbf{k}^{\prime},-\mathbf{k}^{\prime}\right\}$ ) et pour une structure «double-k $»$ (Figure $11 \mathrm{~b} /$ vecteurs de propagation $\{\mathbf{k},-\mathbf{k}\}$ et $\left.\left\{\mathbf{k}^{\prime},-\mathbf{k}^{\prime}\right\}\right)$.

Le paramètre affinable est dans ce cas d'une RI de dimension 1, n'intervenant qu'une fois, le paramètre $\mathrm{C}_{1}$, réel.

\section{$3.3 R_{2} T_{2} X$ : quelques remarques sur la «multiplicité » du vecteur de propagation $k$}

\subsection{1 Étoile de $\boldsymbol{k}$, domaines magnétiques « $\boldsymbol{k} »$}

Par suite de la symétrie quadratique du système étudié, si les vecteurs de propagation $\mathbf{k}$ et $-\mathbf{k}$, où $\mathbf{k}=\left(\mathrm{k}_{\mathrm{x}} 00\right)$, avec $\mathrm{k}_{\mathrm{x}} \neq 0$ et $\mathrm{k}_{\mathrm{x}} \neq 1 / 2$, permettent de décrire la structure magnétique de $\mathrm{Ce}_{2} \mathrm{Pd}_{2} \mathrm{Sn}$, il en est de même des vecteurs $\mathbf{k}^{\prime}$ et $-\mathbf{k}^{\prime}$, où $\mathbf{k}^{\prime}=\left(0 \mathrm{k}_{\mathrm{x}} 0\right)$. Par définition, l'ensemble $\left\{\mathbf{k},-\mathbf{k}, \mathbf{k}^{\prime},-\mathbf{k}^{\prime}\right\}$ est appelé « étoile de $\mathbf{k}$ ». Notons que les différentes branches de cette étoile sont obtenues en appliquant à l'une quelconque d'entre elles, $\mathbf{k}$ par exemple, l'ensemble des opérations du groupe ponctuel de symétrie associé au groupe d'espace du cristal.

Une structure magnétique de $\mathrm{Ce}_{2} \mathrm{Pd}_{2} \mathrm{Sn}$ uniquement définie par $\{\mathbf{k},-\mathbf{k}\}$, ou $\{\mathbf{k},,-\mathbf{k}\}$, est dite « simple-k ». Du point de vue « domaines magnétiques », les solutions « simple-k » sont associées à deux régions différentes de l'espace, l'une « $\mathbf{k} »$, l'autre « $\mathbf{k}$ ' . La figure 11a montre quelles sont alors les positions des pics de Bragg magnétiques autour d'un même nœud du réseau réciproque. Si les deux ensembles, $\{\mathbf{k},-\mathbf{k}\}$ et $\left\{\mathbf{k}^{\prime},-\mathbf{k}^{\prime}\right\}$, interviennent simultanément dans une même région de l'espace du cristal, la structure magnétique (monodomaine) ainsi obtenue est dite « multi-k ». Pour ce dernier cas (structure «double-k» : k et k'), la figure 11b est l'analogue de la figure 11a.

Notons que la diffraction de neutrons sur poudre ne permet pas de trancher entre les deux situations décrites sur la figure $11(11 \mathrm{a}, 11 \mathrm{~b})$, et ce quelle que soit la proportion relative des domaines magnétiques «simple-k». Si par l'application d'une contrainte extérieure il devenait possible de modifier l'équilibre des proportions des domaines magnétiques «simple-k», alors la diffraction de neutrons sur monocristal permettrait de répondre à la question : la structure magnétique « incommensurable » de $\mathrm{Ce}_{2} \mathrm{Pd}_{2}$ In est-elle «simple» ou « multi-k »?

\subsubsection{Structure magnétique $U_{2}\left(N i_{1-x} P d_{x}\right)_{2} S n$ : plusieurs vecteurs de propagation pour un même composé}

Les structures magnétiques des composés extrêmes de la solution solide $\mathrm{U}_{2}\left(\mathrm{Ni}_{1-\mathrm{x}} \mathrm{Pd}_{\mathrm{x}}\right)_{2} \mathrm{Sn}$ sont représentées sur la figure 1 : vecteur de propagation $\mathbf{k}=\left(\begin{array}{ll}0 & 0\end{array}\right)$ et moments magnétiques perpendiculaires à l'axe quadratique pour $\mathrm{x}=1, \mathrm{U}_{2} \mathrm{Pd}_{2} \mathrm{Sn}$ [Figure 1a]; vecteur de propagation $\mathbf{k}=(001 / 2)$ et moments magnétiques parallèles à l'axe c pour $\mathrm{x}=0, \mathrm{U}_{2} \mathrm{Ni}_{2} \mathrm{Sn}$ [Figure 1d]. Nous avons choisi d'étudier la 


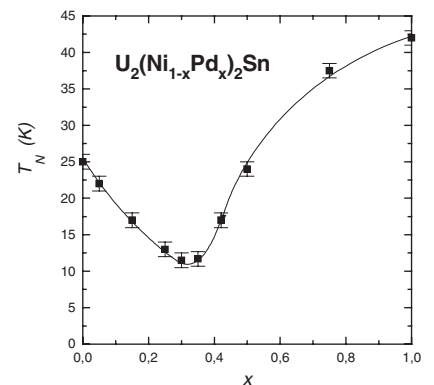

Figure 12. $\mathrm{U}_{2}\left(\mathrm{Ni}_{1-\mathrm{x}} \mathrm{Pd}_{\mathrm{x}}\right)_{2} \mathrm{Sn}$ : variation de la température de Néel $\left(\mathrm{T}_{\mathrm{N}}\right)$ en fonction de la composition $(\mathrm{x})$.
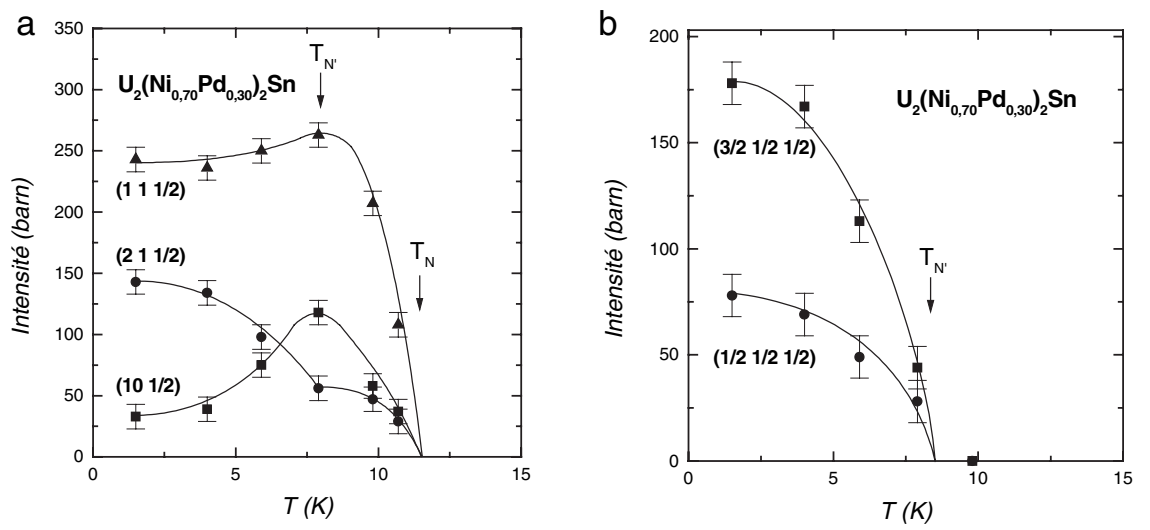

Figure 13. $\mathrm{U}_{2}\left(\mathrm{Ni}_{0.70} \mathrm{Pd}_{0.30}\right)_{2} \mathrm{Sn}$ : suivi thermique des raies magnétiques associées aux vecteur de propagation $\left(\begin{array}{lll}0 & 1 / 2\end{array}\right)$ [Figure 13a] et $(1 / 21 / 21 / 2)$ [Figure 13b].

solution solide $\mathrm{U}_{2}\left(\mathrm{Ni}_{1 \mathrm{x}} \mathrm{Pd}_{\mathrm{x}}\right)_{2} \mathrm{Sn}[1,15]$, afin de suivre l'évolution des structures magnétiques en fonction de la composition, en termes de vecteur de propagation (échange RKKY) et direction des moments magnétiques (anisotropie).

Étape préalable à toute étude de structure(s) magnétique(s), les mesures de susceptibilité magnétique ont permis de montrer que tous ces composés étaient antiferromagnétiques à basse température, la température de Néel $\mathrm{T}_{\mathrm{N}}$ de la série (Figure 12) étant minimale pour $0.30 \leq \mathrm{x} \leq 0.35$.

Les diagrammes de diffraction de neutrons associés au composé de $\mathrm{T}_{\mathrm{N}}$ minimale $\mathrm{U}_{2}\left(\mathrm{Ni}_{0.70} \mathrm{Pd}_{0.30}\right)_{2} \mathrm{Sn}$ montrent que deux vecteurs de propagation, non reliés par une opération de symétrie cristalline, sont nécessaires à l'indexation de toutes les raies de Bragg magnétiques présentes à $\mathrm{T}<\mathrm{T}_{\mathrm{N}}$ : $\mathbf{k}_{1}=\left(\begin{array}{lll}0 & 1 / 2\end{array}\right)$ d'une part et $\mathbf{k}_{2}=(1 / 21 / 21 / 2)$ d'autre part. La figure 13 permet de suivre l'évolution thermique des principales raies magnétiques de chaque catégorie, respectivement associées aux vecteurs de propagation $\mathbf{k}_{1}$ [Figure 13a] et $\mathbf{k}_{2}$ [Figure 13b].

Trois domaines de température sont donc observés du point de vue des propriétés magnétiques :

1. Au-dessus de $\mathrm{T}_{\mathrm{N}}=11,5(1) \mathrm{K}, \mathrm{U}_{2}\left(\mathrm{Ni}_{0.70} \mathrm{Pd}_{0.30}\right)_{2} \mathrm{Sn}$ est paramagnétique.

2. Entre $\mathrm{T}_{\mathrm{N}^{\prime}}=8,0(1) \mathrm{K}$ et $\mathrm{T}_{\mathrm{N}}$, un seul vecteur de propagation $\mathbf{k}_{1}=(001 / 2)$ est nécessaire à la description de la structure magnétique. L'analyse des intensités magnétiques du diagramme de diffraction de neutrons conduit à une structure de type $\mathrm{U}_{2} \mathrm{Ni}_{2} \mathrm{Sn}$ [Figure $1 \mathrm{~d}$ ].

3. Au-dessous de $\mathrm{T}_{\mathrm{N}^{\prime}}$, deux vecteurs de propagation sont nécessaires à l'indexation du diagramme de

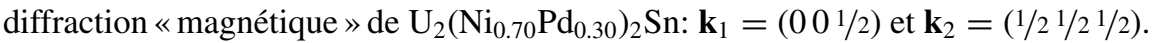

L'analyse des intensités magnétiques « $\mathbf{k}_{2}=(1 / 21 / 21 / 2) »$ conduit à une structure où les moments magnétiques d'une même cellule élémentaire sont tous identiques et parallèles à l'axe c. Ce résultat ne fait intervenir qu'une seule RI. La liste de ces RI n'est pas donnée dans ce texte, mais le lecteur 

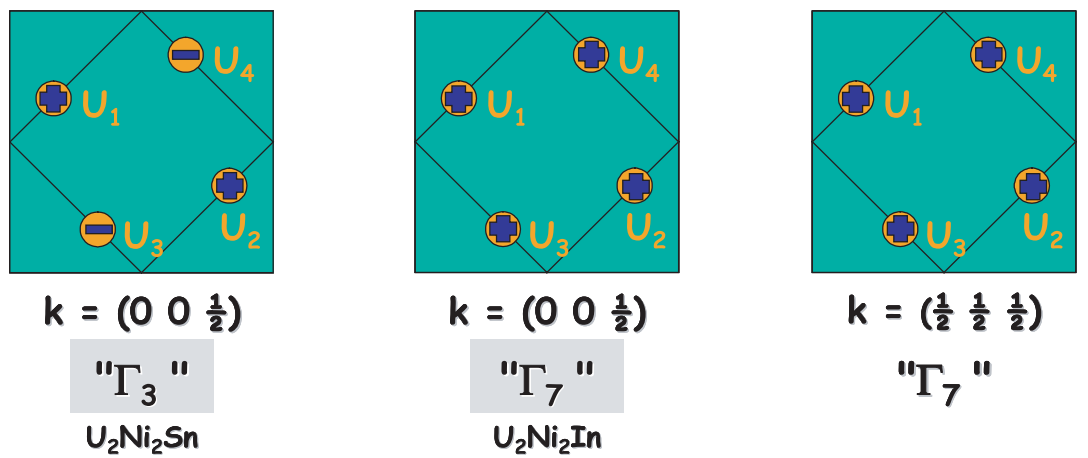

Figure 14. Représentation graphique des structures magnétiques de $\mathrm{U}_{2}\left(\mathrm{Ni}_{0.70} \mathrm{Pd}_{0.30}\right)_{2} \mathrm{Sn}$. Les schémas ci-dessus ne représentent que les moments magnétiques des atomes d'une même cellule élémentaire. Pour un vecteur de propagation $\mathbf{k}$, le déphasage entre deux moments magnétiques distants de $\mathbf{G}=\mathrm{n}_{1} \mathbf{a}+\mathrm{n}_{2} \mathbf{b}+\mathrm{n}_{3} \mathbf{c}\left(\mathrm{n}_{1}, \mathrm{n}_{2}, \mathrm{n}_{3}\right.$ entiers relatifs) est égal au produit scalaire $2 \pi \mathbf{k} \cdot \mathbf{G}$. Les notations " $\Gamma_{i}$ " renvoient à la figure 7 . Les structures magnétiques $\mathbf{k}=\left(\begin{array}{lll}0 & 0 & 1 / 2\end{array}\right)$ de $\mathrm{U}_{2} \mathrm{Ni}_{2} \mathrm{Sn}$ et $\mathrm{U}_{2} \mathrm{Ni}_{2} \mathrm{In}$ sont représentées sur la figure 1 .

se convaincra aisément, via BASIREPS, que les structures magnétiques associées sont, dans une maille élémentaire, identiques à celles obtenues dans le cas $\mathbf{k}=\left(\begin{array}{lll}0 & 0 & 0\end{array}\right)$ analysé en $\mathbf{3 . 1}$. Seule « différence », le passage d'une cellule élémentaire à une cellule voisine s'accompagne systématiquement pour « $\mathbf{k}_{2}=$ $(1 / 21 / 21 / 2) \gg$ d'un changement du signe des moments, quelle que soit la translation envisagée, a, b ou c.

L'analyse des intensités magnétiques « $\mathbf{k}_{1}=\left(\begin{array}{lll}0 & 0 & 1 / 2\end{array}\right) »$ fait intervenir, au-dessous de $\mathrm{T}_{\mathrm{N}^{\prime}}, 2 \mathrm{RI}$ différentes (même remarque que ci-dessus en ce qui concerne la liste de ces RI) et conduit ainsi à deux

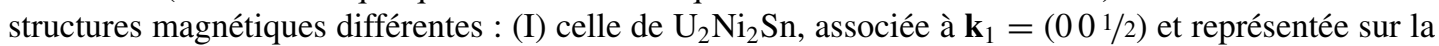
figure $1 \mathrm{~d}$; (II) celle, toujours associée à $\mathbf{k}_{1}=\left(\begin{array}{lll}0 & 1 & 1 / 2\end{array}\right)$, mais pour laquelle les moments magnétiques d'une même cellule élémentaire sont tous identiques [Figure 1c].

$\mathrm{Au}$-dessous de $\mathrm{T}_{\mathrm{N}^{\prime}}$, une question reste cependant posée, à laquelle la diffraction de neutrons ne permet pas de répondre : les trois «structures magnétiques » décrites ci-dessus sont-elles associées à 3 zones volumiques différentes d'un même cristal ou correspondent-elles, dans l'hypothèse inverse, à un même volume physique?

Si nous retenons l'option de structure(s) magnétique(s) ne possédant que des moments de même grandeur, la seule manière d'y parvenir est d'imaginer trois volumes différents (domaines magnétiques), pour trois structures magnétiques différentes. Ces structures magnétiques sont représentées sur la figure 14. Ainsi, à T = 1.5 K, avons-nous obtenu, dans l'hypothèse supplémentaire d'une norme unique pour les moments magnétiques associés à chaque domaine, les pourcentages volumiques suivants :

$$
\begin{aligned}
& \text { « } \mathbf{k}_{1}=(00,1 / 2) » \quad 30(5) \% \quad \text { Structure magnétique de type } \mathrm{U}_{2} \mathrm{Ni}_{2} \mathrm{Sn} \text { (I) } \\
& 《 \mathbf{k}_{1}=\left(\begin{array}{lll}
0 & 0 & 1 / 2
\end{array}\right) » \quad 25(5) \% \quad \text { Structure magnétique de type } \mathrm{U}_{2} \mathrm{Ni}_{2} \mathrm{In} \text { (II) } \\
& \ll \mathbf{k}_{2}=(1 / 21 / 21 / 2) » 45(5) \% \text {. }
\end{aligned}
$$

\section{COMMENTAIRES}

\subsection{Transitions de phase : théorie de Landau, symétrie et représentations irréductibles}

La théorie de Landau des transitions de phase d'ordre 2 [16, 17] permet d'exprimer l'énergie libre d'un système physique au voisinage de la température de transition $\mathrm{T}_{\mathrm{C}}$ sous la forme d'un développement limité :

$$
\mathrm{F}(\mathrm{T}, \eta)=\mathrm{F}(\mathrm{T}, 0)+\alpha\left(\mathrm{T}_{\mathrm{C}}-\mathrm{T}\right) \eta^{2}+\beta \eta^{4}+\ldots
$$


avec $\alpha$ et $\beta$ paramètres indépendants de la température, et respectivement $<0$ et $>0$. Dans cette expression, le paramètre $\eta$ est le paramètre d'ordre de la transition. L'existence de ce paramètre est liée à la notion de «brisure de symétrie », la phase désordonnée du système considéré $\left(\mathrm{T}>\mathrm{T}_{\mathrm{C}}\right)$ étant invariante par un groupe de transformations $G$, et la phase ordonnée $\left(\mathrm{T}<\mathrm{T}_{\mathrm{C}}\right)$ ne l'étant que sous l'action d'un sous-groupe du groupe $G$. Le paramètre d'ordre, qui caractérise la transition, est ainsi une quantité invariante sous l'action du sous-groupe, mais pas du groupe $G$ tout entier. Ce paramètre, continu à $\mathrm{T}_{\mathrm{C}}$, est ainsi nul au-dessus de $\mathrm{T}_{\mathrm{C}}$ et $\neq 0$ au-dessous.

Le paramètre d'ordre qui caractérise une transition paramagnétique/ferromagnétique est l'aimantation du système, supposée uniforme, soit $\eta=\mathrm{M}$. Dans le cas des composés 2:2:1 considérés dans les paragraphes précédents, et pour une structure ferromagnétique de moments parallèles à l'axe quadratique, caractérisée donc par le vecteur de propagation $\mathbf{k}=\left(\begin{array}{lll}0 & 0 & 0\end{array}\right)$ et la Représentation Irréductible (RI) $\Gamma_{7}$ de dimension 1 (voir Figure 7), ce paramètre devient $\eta=\left(M_{1 z}+M_{2 z}+M_{3 z}+M_{4 z}\right)$. Notons que si le passage de $M$ à $\left(M_{1 z}+M_{2 z}+M_{3 z}+M_{4 z}\right)$ paraît évident en termes d'aimantation du système, $\left(M_{1 z}+M_{2 z}+M_{3 z}+M_{4 z}\right)$ représente aussi le « vecteur de base » associée à la RI $\Gamma_{7}$.

De manière plus générale, le paramètre d'ordre associé à l'une quelconque des structures magnétiques de la figure 7 est le vecteur de base de la RI considérée : ainsi la structure magnétique de $\mathrm{U}_{2} \mathrm{Pd}_{2} \mathrm{In}$ (voir section 3) est-elle associée au paramètre $\eta=\left(\mathrm{M}_{1 \mathrm{x}}-\mathrm{M}_{2 \mathrm{x}}+\mathrm{M}_{3 \mathrm{x}}-\mathrm{M}_{4 \mathrm{x}}\right)+\left(\mathrm{M}_{1 \mathrm{y}}-\right.$ $\left.\mathrm{M}_{2 \mathrm{y}}+\mathrm{M}_{3 \mathrm{y}}-\mathrm{M}_{4 \mathrm{y}}\right)$.

Dire que pour une transition magnétique continue (ordre 2), un seul paramètre d'ordre devient critique à $\mathrm{T}_{\mathrm{C}}$, c'est dire que la structure magnétique qui s'établit au-dessous de la température d'ordre du système ne peut faire intervenir qu'un seul ensemble de vecteurs de base, celui associé à une même Représentation Irréductible $\Gamma_{\mathrm{i}}$. La technique d'analyse séquentielle des sous-ensembles de vecteurs de base proposés par l'analyse de symétrie (RI) trouve ainsi sa justification. On notera cependant que la structure magnétique, qui a été obtenue à $\mathrm{T}=1.5 \mathrm{~K}$ pour le système $\mathrm{U}_{2}\left(\mathrm{Ni}_{0.70} \mathrm{Pd}_{0.30}\right)_{2} \mathrm{Sn}$, fait appel pour le même vecteur de propagation $\mathbf{k}=\left(\begin{array}{lll}0 & 0 & 1 / 2\end{array}\right)$, à deux représentations irréductibles distinctes, " $\Gamma_{3}$ " et " $\Gamma_{7}$ ".

\subsection{Diffraction de neutrons : sur quels diffractomètres ?}

Comme rappelé dans l'introduction, la diffraction de neutrons fait intervenir de manière «parallèle»facteur de structure nucléaire $\mathrm{F}_{\mathrm{N}}(\mathbf{K})$ [scalaire] et facteur de structure magnétique $\mathbf{F}_{\mathrm{M}}(\mathbf{K})$ [vecteur] au travers de sa composante perpendiculaire au vecteur de diffusion, $\mathbf{F}_{\perp \mathrm{M}}(\mathbf{K})$ (vecteur d'interaction magnétique). Si le faisceau de neutrons incidents est non polarisé, l'intensité totale mesurée est proportionnelle à la somme des intensités nucléaires et magnétiques, soit $\left\{\mathrm{F}_{\mathrm{N}}(\mathbf{K})\right\}^{2}+\left\{\mathbf{F}_{\perp \mathrm{M}}(\mathbf{K}) \cdot \mathbf{F}^{*} \perp \mathrm{M}(\mathbf{K})\right\}$.

Les amplitudes de diffusion nucléaires et magnétiques sont, on l'a vu, du même ordre de grandeur $\left(10^{-12} \mathrm{~cm}\right)$. Cependant, si la valeur de la première de ces amplitudes, d'origine nucléaire, est indépendante du vecteur de diffusion $\mathbf{K}$, il n'en est pas de même pour la seconde, dont l'expression, $\left(\gamma \mathrm{r}_{0} / 2\right) \mathrm{f}(\mathrm{K}) \mathbf{M}_{\perp} \cdot \mathbf{S}$, fait intervenir $\mathrm{f}(\mathbf{K})$, facteur de forme magnétique de l'atome (ion) considéré. Ce facteur de forme est l'analogue de celui observé en diffraction X, à la différence que la transformée de Fourier est maintenant celle de la densité des seuls électrons non appariés. La figure 15 reproduit pour l'ion $\mathrm{U}^{3+}$ la variation du facteur de forme en fonction de $\sin \theta / \lambda$.

La conséquence immédiate de cette variation est que les contributions magnétiques aux pics de Bragg seront plus intenses aux faibles valeurs de $\sin \theta / \lambda$, et qu'un diffractomètre «poudres » adapté à la détermination des structures magnétiques se doit d'avoir une courbe de résolution telle que la largeur des pics soit minimale aux faibles valeurs de $\sin \theta / \lambda$. La figure 16 présente l'évolution du paramètre de largeur à mi-hauteur (FWHM) d'origine instrumentale pour les diffractomètres 3T2 (neutrons thermiques) et G4.1 (neutrons froids) du LLB. Ce dernier diffractomètre (haut flux, moyenne résolution) est ainsi celui qui doit être préférentiellement utilisé pour l'étude des structures magnétiques. 


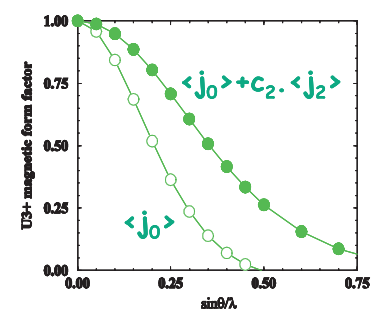

$f(\sin \theta / \lambda) / U^{3+}$ magnetic form factor

Figure 15. Facteur de forme magnétique de l'ion $\mathrm{U}^{3+}$ (approximation dipolaire, fonctions $\left\langle\mathrm{j}_{0}\right\rangle$ et $\left\langle\mathrm{j}_{2}\right\rangle$ : calcul relativiste de Desclaux et Freeman [18]).

a

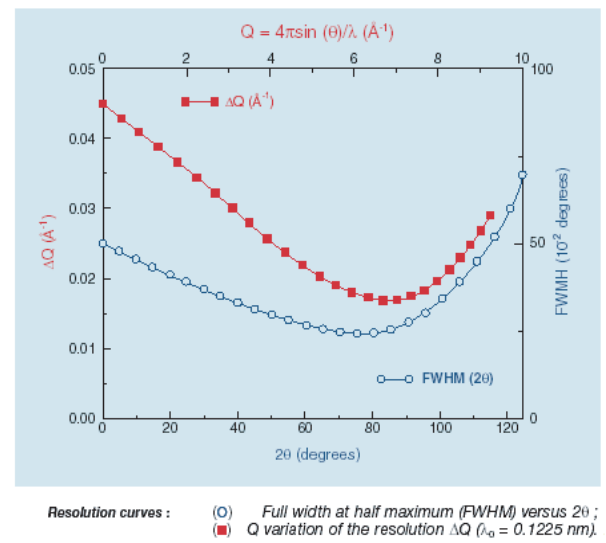

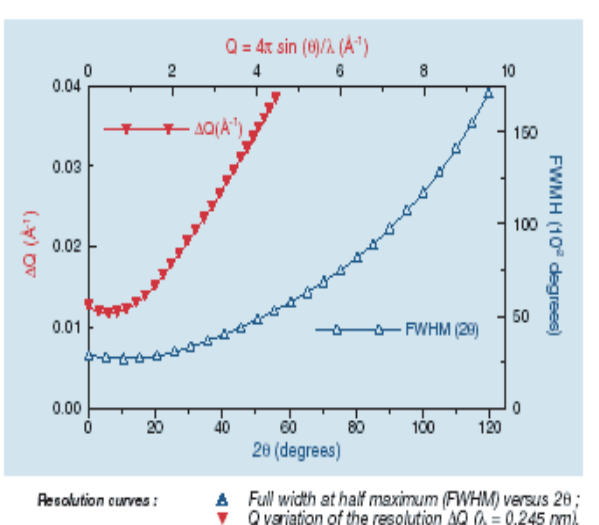

Figure 16. Caractéristiques géométriques des spectromètres 2-axes du LLB, 3T2 (Figure 16a) et G4.1 (Figure 16b) : «WHM » (Full Width at Half Maximum) en fonction de l'angle $2 \theta$ (symboles ouverts) et $\Delta \mathrm{Q}$ en fonction du vecteur de diffusion $Q=4 \pi \sin \theta / \lambda$ (symboles pleins).

A l'ILL, les 2-axes D20 et D1B (schéma ci-dessous) sont les mieux adaptés. Nous renvoyons aux sites de l'ILL [www.ill.fr], du LLB [www-llb.cea.fr], et plus généralement au portail [neutron.neutroneu.net], qui donne accès à la description des caractéristiques des spectromètres pour l'ensemble des réacteurs européens.

\section{3 $\mathbf{R}_{2} \mathrm{~T}_{2} \mathrm{X}$, diffraction sur monocristal}

La première structure magnétique publiée dans la série 2:2:1 [2] est celle de $\mathrm{U}_{2} \mathrm{Ni}_{2} \mathrm{Sn}$ : structure magnétique colinéaire, de vecteur de propagation $\mathbf{k}=\left(\begin{array}{lll}0 & 0 & 1\end{array}\right.$ étant perpendiculaires à l'axe quadratique c. Ce résultat a été obtenu par l'analyse de données de diffraction de neutrons sur poudre : si la solution retenue correspond au «meilleur»affinement des données expérimentales, elle n'obéit pas à la règle empirique du $§ 4.4$. Nous avons donc repris cette étude ancienne, mais sur un monocristal (F. WASTIN, Institute for Transuranium Elements, Karlsruhe). L'expérience a été réalisée à l'ILL (D15- diffractomètre «bras levant » - $\lambda=1.17 \AA$ ), et montre sans ambiguïté [8] que la structure magnétique de $\mathrm{U}_{2} \mathrm{Ni}_{2} \mathrm{Sn}$ est bien celle qui avait été proposée du point de vue disposition relative des différents moments magnétiques dans la maille cristalline, mais que ces moments sont parallèles à l'axe quadratique c, axe de facile aimantation. La mesure de diffraction sur monocristal a en effet permis d'accéder à un nombre de raies magnétiques supérieur à celui de

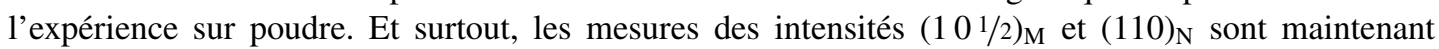
indépendantes. 


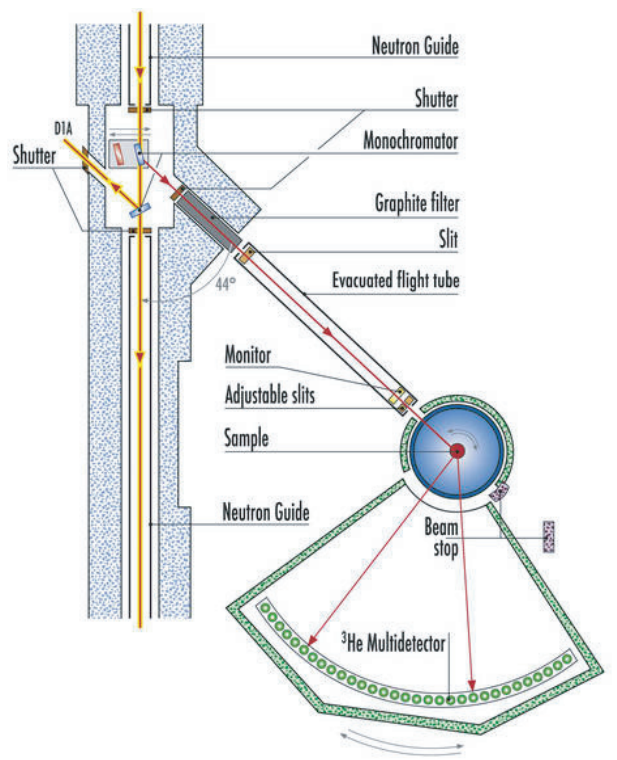

Figure 17. Diffractomètre 2-axes D1B (ILL).
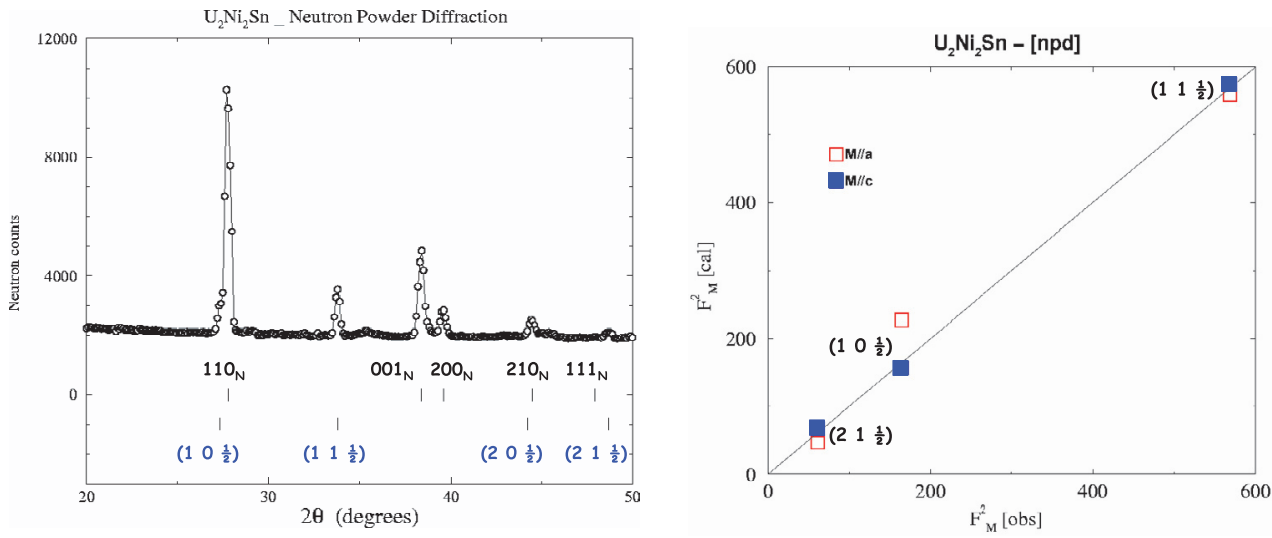

Figure 18. $\mathrm{U}_{2} \mathrm{Ni}_{2} \mathrm{Sn}$. A gauche de la figure, diagramme de diffraction de neutrons sur poudre mesuré à $\mathrm{T}=$ $1.5 \mathrm{~K}(\mathrm{G} 4.1-\lambda=2.425 \AA$ ) : points expérimentaux (symboles), et positions des pics de Bragg Nucléaires (rangée supérieure) et Magnétiques (rangée inférieure). A droite diffraction de neutrons sur monocristal : comparaison des intensités magnétiques observées et calculées, pour les raies $\left(10 \frac{1}{1} / 2\right),\left(11 \frac{1}{1} 2\right)$ et $\left(21 \frac{1}{1 / 2}\right)$ et les directions de moments magnétiques $\mathbf{M} / / \mathbf{a}$ (symboles ouverts) et $\mathbf{M} / / \mathbf{c}$ (symboles fermés). La structure magnétique de $\mathrm{U}_{2} \mathrm{Ni}_{2} \mathrm{Sn}$ $(\mathbf{M} / / \mathbf{c})$ est représentée sur la figure 1.

\section{4 $\mathbf{R}_{2} \mathbf{T}_{2} \mathrm{X}$ et structures magnétiques : quelques pistes d'interprétation}

Les premières déterminations de structures magnétiques ont eu lieu, pour les intermétalliques de stoechiométrie 2:2:1, au début de la décennie 90'. De nombreux résultats ont ainsi été publiés, par des équipes différentes, sur les composés stoechiométriques $\mathrm{R}_{2} \mathrm{~T}_{2} \mathrm{X}\{\mathrm{R}=\mathrm{U}, \mathrm{Ce}, \mathrm{Nd}, \mathrm{Tb}, \mathrm{Dy}, \mathrm{Ho}$, $\mathrm{Er} / \mathrm{T}=\mathrm{Fe}, \mathrm{Co}, \mathrm{Ni}, \mathrm{Ru}, \mathrm{Rh}, \mathrm{Pd} / \mathrm{X}=\mathrm{In}, \mathrm{Sn}\}$ et les solutions solides $\mathrm{U}_{2} \mathrm{Pd}_{2+\mathrm{x}} \mathrm{Sn}_{1-\mathrm{x}}, \mathrm{Ce}_{2} \mathrm{Pd}_{2+\mathrm{x}} \mathrm{Sn}_{1-\mathrm{x}}$ et $\mathrm{Ce}_{2} \mathrm{Pd}_{2+x} \mathrm{In}_{1-\mathrm{x}}$ dont la structure cristalline est de type $\mathrm{U}_{2} \mathrm{Fe}_{2} \mathrm{Sn}$ (symétrie quadratique, de groupe d'espace $\mathrm{P} 4 / \mathrm{mbm})$. 
Comme indiqué ci-dessus (\$2.2), les structures magnétiques qui ont été obtenues sont extrêmement variées, tant du point de vue vecteur de propagation qu'arrangement relatif des moments magnétiques $\mathbf{M}_{1}, \mathbf{M}_{2}, \mathbf{M}_{3}$ et $\mathbf{M}_{4}$ au sein de la maille cristalline unité. Une règle (empirique) existe, qui lie la direction de la liaison U-U (R-R) la plus courte et celles des moments magnétiques $\mathbf{M}_{1-4}$, de la structure ordonnée. Le tableau suivant donne ainsi, en fonction des paramètres cristallins $\mathrm{a}, \mathrm{c}$ et du paramètre de position $\mathrm{x}_{\mathrm{U}}$, l'expression des distances des premiers voisins $\mathrm{U}-\mathrm{U}$, les directions de ces liaisons $(/ / \mathbf{c}$ ou $\perp \mathbf{c})$ et la symbolique qui leur est associée.

\begin{tabular}{|c|c|c|c|}
\hline Nombre des voisins & 1 & 2 & 4 \\
\hline Distance U-U & $\mathrm{d}_{1 \mathrm{U}}=2 \sqrt{2} \mathrm{x}_{\mathrm{U}} \mathrm{a}$ & $\mathrm{d}_{2 \mathrm{U}}=\mathrm{c}$ & $\mathrm{d}_{4 \mathrm{U}}=\mathrm{a} \sqrt{(1 / 2)^{2}+\left(1 / 2-x_{\mathrm{U}}\right)^{2}}$ \\
\hline Direction U-U & $\perp \mathbf{c}$ & $/ / \mathbf{c}$ & $\perp \mathbf{c}$ \\
\hline $\mathbf{M}_{1}, \mathbf{M}_{2}, \mathbf{M}_{3}, \mathbf{M}_{4}$ & $/ / \mathbf{c}$ & $\perp \mathbf{c}$ & \\
\hline Symbolique (Figure 19) & \{pointillés & & $\{$ trait continu $\}$ \\
\hline
\end{tabular}

Sont aussi données sur ce même tableau les directions des moments magnétiques des structures magnétiques observées : ces moments sont respectivement parallèles et perpendiculaires à l'axe quadratique selon que la plus courte liaison U-U correspond à des atomes d'un même plan $(\mathbf{a}, \mathbf{b})$ [distance $\mathrm{d}_{1 \mathrm{U}}$ ] ou à des atomes séparés de $\mathbf{c}$ [distance $\mathrm{d}_{2 \mathrm{U}}$ ].

Pour ces composés intermétalliques, un modèle d'interactions magnétiques de type RKKY [Rudermann-Kittel-Kasuya-Yosida], respectant la symétrie quadratique des systèmes étudiés, a été développé, qui a permis de rendre compte des structures magnétiques $\mathbf{k}=\left(\begin{array}{lll}0 & 0 & 0\end{array}\right), \mathbf{k}=\left(\begin{array}{lll}0 & 0 & 1 / 2\end{array}\right), \mathbf{k}=$ $(1 / 21 / 20)$ et $\mathbf{k}=(1 / 21 / 21 / 2)$ observées pour les systèmes $(\mathrm{U}, \mathrm{Ce})_{2} \mathrm{Pd}_{2} \mathrm{Sn}, \mathrm{U}_{2} \mathrm{Pd}_{2+\mathrm{x}} \mathrm{Sn}_{1-\mathrm{x}}$ et $\mathrm{U}_{2}(\mathrm{Ni}, \mathrm{Pd})_{2} \mathrm{Sn}$. Les paramètres de ce modèle uniaxial, et non plus sphérique, sont les composantes $\mathrm{k}_{\mathrm{F}}$ et $\mathrm{k}_{\mathrm{Fz}}$ du vecteur de Fermi dans les directions perpendiculaires et parallèles à l'axe c. Nous renvoyons à l'article original [19], pour le tracé des diagrammes de phase $\mathrm{k}_{\mathrm{F}}, \mathrm{k}_{\mathrm{Fz}}$ à $\mathrm{T}=0 \mathrm{~K}$ (état fondamental).

Du point de vue modélisation, il convient de ne pas oublier l'existence des programmes SIMBO et ENERMAG [www.ill.fr/sites/fullprof/..], développés dans le but d'interpréter, voire de prévoir, les structures magnétiques expérimentales des isolants, dans le cas où celles-ci sont obtenues via des interactions magnétiques de super- et/ou super-super-échange. Dans une première étape, le programme SIMBo analyse la structure cristalline en termes de chemins de super-échange $\mathrm{M}_{1}-\mathrm{X}-\mathrm{M}_{2}$ et de supersuper-échange $\mathrm{M}_{1}-\mathrm{X}_{1}-\mathrm{X}_{2}-\mathrm{M}_{2}$ à partir de la connaissance du groupe d'espace, des coordonnées atomiques dans l'unité asymétrique, des charges ioniques, des moments magnétiques et des paramètres de maille. Dans une deuxième étape, en utilisant les interactions issues de sIMBo, le programme ENERMAG permet d'obtenir un(des) diagramme(s) de phase magnétiques en fonction des paramètres d'échange (modèle isotrope). Les publications [20-24] sont ainsi associées aux diagrammes de phases

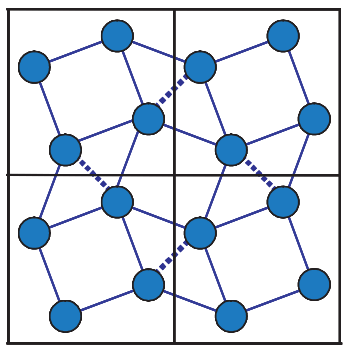

Figure 19. Structure cristalline $2: 2: 1(\mathrm{P} 4 / \mathrm{mbm})$ et distances $U-U$ dans le plan a-b: $d_{1 U}$ (pointillés) et $d_{4 U}$ (traits continus). 
magnétiques de $\mathrm{MFePO}_{5}$ [20], $\mathrm{CuFe}_{2}\left(\mathrm{P}_{2} \mathrm{O}_{7}\right)_{2}$ [21], $\mathrm{Li}_{3} \mathrm{Fe}_{2}\left(\mathrm{PO}_{4}\right)_{3}$ [22], $\mathrm{LiFe}\left(\mathrm{P}_{2} \mathrm{O}_{7}\right)$ [23] et $\mathrm{RMn}_{2} \mathrm{O}_{5}$ [24] obtenus dans le cadre de ce modèle.

\section{CONCLUSION}

La diffraction de neutrons est à l'heure actuelle, et restera encore pour un bon nombre d'années, la technique la plus directe de détermination des structures magnétiques. Dans ce document, nous avons présenté les étapes successives d'une telle détermination structurale (structures ferromagnétiques, antiferromagnétiques, structures magnétiques commensurables, incommensurables) pour un ensemble de composés de même structure cristalline : $\mathrm{U}_{2} \mathrm{~T}_{2} \mathrm{X}$, avec $\mathrm{T}=$ Métal de Transition et $\mathrm{X}=\mathrm{In}$ ou $\mathrm{Sn}$.

Dans cet article, une attention particulière a été réservée à la notion de symétrie [25-26] et à l'analyse de cette symétrie dans le cadre de la «théorie des représentations de Bertaut » [27]. Nous avons détaillé deux cas, associés à des structures magnétiques respectivement commensurable et incommensurable. Les résultats que nous avons présentés ont utilisé le programme BASIREPS, accessible via FulLProf. D'autres programmes existent : MoDY, SARAH, que nous ne présenterons pas ici. Nous renvoyons aux publications originales de leurs auteurs [28, 29].

Dans cet article nous n'avons pas particulièrement détaillé l'étape suivante de détermination d'une structure magnétique : la comparaison des données expérimentales de diffraction et des modèles de structures magnétiques potentielles. Encore une fois, les résultats présentés ici ont été acquis via FullProf. Ce logiciel fait partie d'un ensemble de programmes [FullProf_Suite] qui ont été développés par l'un des auteurs de cette contribution [JRC] dans un cadre extrêmement général, pour le traitement des données de diffraction, que ces données aient été obtenues sur poudre et/ou monocristal, par diffraction de RX et/ou de neutrons, que ces neutrons enfin soient issus d'une source continue et/ou pulsée. Cette «suite» est disponible sur le site de l'ILL (http://www.ill.fr/sites/fullprof/...), sous «format » Windows, Linux ou Mac OS.

La détermination des structures magnétiques fait essentiellement appel à cinq des programmes inclus dans FullProf_Suite : Basireps, FullProf, FP_Studio, K_SEArCh et WinPlotr.

WinPLOTR est indispensable à la visualisation des diagrammes de diffraction, et la multiplicité des options accessibles ne sera pas évoquée ici.

K_SEARCH est un programme qui permet de déterminer le(s) vecteur(s) de propagation de la structure magnétique (k) à partir des données (position des raies de Bragg magnétiques) d'un diagramme de diffraction sur poudre mesuré au-dessous de la température de transition $T_{C}\left(T_{N}\right)$ et de la métrique associée à la structure cristalline du composé étudié.

BASIREPS, on l'a vu, permet de calculer les vecteurs de base des RI. Ses résultats peuvent être directement insérés dans le fichier de contrôle de FulLProf (fichier.pcr). Au delà de l'option «classique» d'analyse par moindres carrés du profil du diagramme de diffraction poudre enregistré, il existe dans FullProf une option d'affinement de structure magnétique qui utilise l'algorithme de «recuit simulé »décrit en $[30,31]$. L'utilisation de ce processus est recommandée dès que le nombre de paramètres devient important, en particulier lorsque l'analyse de symétrie ne fournit pas de contraintes suffisantes entre ces paramètres. La qualité des données expérimentales est bien sûr essentielle au succès de l'opération.

Enfin, FP_STUdio permet de visualiser n'importe quelle structure magnétique, qu'elle soit commensurable (Figure 1) ou incommensurable (Figure 10), colinéaire (Figure 1, Figure 10) ou non (Figure 1).

Les expériences de diffraction de neutrons qui ont été analysées ici sont essentiellement des expériences de diffraction sur poudres. Ces expériences sont nécessairement les premières à réaliser dans le cadre d'une détermination de structure magnétique, mais dans certains cas les données obtenues peuvent ne pas être suffisantes pour lever toutes les ambiguïtés de cette détermination de structure. Un exemple a été donné $\left(\mathrm{U}_{2} \mathrm{Ni}_{2} \mathrm{Sn}, \S 4.3\right)$, où la diffraction sur monocristal a clairement permis de trancher entre deux structures magnétiques associées à deux RI différentes (" $\Gamma_{10}$ " et " $\Gamma_{3}$ "). 
Ce travail de diffraction de neutrons sur les intermétalliques «2:2:1» a été réalisé en collaboration avec l'Institut de Chimie de la Matière Condensée de Bordeaux [ICMCB], dans le cadre de la thèse de doctorat de D. Laffargue [CEA-Région Aquitaine]. L'un des auteurs [FB] voudrait remercier ici tout particulièrement B. Chevalier [ICMCB], pour ces années de fructueuse collaboration et souligner aussi le rôle fondamental des laboratoires de Chimie du Solide pour la «conception » et la préparation de nouveaux matériaux. La première publication sur la «famille 2:2:1 » est en effet issue de l'ICMCB [32].

Dernière remarque : cette présentation est loin d'être exhaustive, aussi ne saurions-nous oublier ici l'article de revue de J. Rossat-Mignod [33] sur le sujet. Ainsi que les articles de J. Schweizer [34] et A. Wills [35], accessibles sur le site d'EDP Sciences.

\section{Références}

[1] Laffargue D., «Structures magnétiques de nouveaux stannures ternaires à base d'uranium ou de terres rares $\mathrm{T}_{2} \mathrm{M}_{2} \mathrm{Sn}(\mathrm{T}=\mathrm{U}, \mathrm{RE}$ et $\mathrm{M}=\mathrm{Ni}, \mathrm{Pd}) »$, Doctorat, Université Bordeaux, Juillet, 1997.

[2] Bourée F., Chevalier B., Fournes L., Mirambet F., Roisnel T., Tran V. H. and Zolnierek Z., JMMM 138, 307 (1994).

[3] Purwanto A., Robinson R. A., Havela L., Sechovsky V., Svoboda P., Nakotte H., Prokes K., de Boer F. R., Seret A., Winand J. M., Rebizant J. and Spirlet J. C., Phys. Rev. B 50, 6792 (1994).

[4] Tran V. H., Zolnierek Z., Bourée F. and Roisnel T., JMMM 161, 270 (1996).

[5] Nakotte H., Purwanto A., Robinson R. A., Prokes K., Klaasse J. C. P., de Chatel P. F., de Boer F. R., Havela L., Sechovsky V., Pereira L. C., Seret A., Rebizant J., Spirlet J. C. and Trouw F., Phys. Rev. B 53, 3263 (1996).

[6] Pereira L. C., Paixao J. A., Estrela P., Godinho M., Bourdarot F., Bonnet M., Rebizant J., Spirlet J. C. and Almeida M., J. Phys: Condens. Matter 8, 11167 (1996).

[7] Martin-Martin A., Pereira L. C., Lander G. H., Rebizant J., Wastin F., Spirlet J. C., Dervenagas P. and Brown P. J., Phys. Rev. B 59, 11818 (1999).

[8] Bourée F., Laffargue D., Chevalier B., Boucherle J. X., Bourdarot F., Burlet P., Rebizant J. and Wastin F., Journées des Actinides (St-Malo, France, 2001).

[9] Laffargue D., Fourgeot F., Bourée F., Chevalier B., Roisnel T. and Etourneau J., Solid State Communications 100, 575 (1996).

[10] Izyumov Y. A., Ozerov R. P., «Magnetic Neutron Diffraction» (Plenum Press, New York, 1970).

[11] Craknell A. P., « Magnetism in Crystalline Materials» (Pergamon Press, Oxford, 1975).

[12] Joshua S. J., «Symmetry Principles and Magnetic Symmetry in Solid State Physics», Adam Hilger, Bristol (1991).

[13] Kovalev O. V., «Representations of the Crystallographic Space Groups: Irreducible Representations and Corepresentations », $2^{\text {nd }}$ Edition, Gordon and Breach Science Publishers, Switzerland (1993).

[14] Shubnikov A. V. and Belov N. V., «Colored Symmetry », Pergamon Press, Oxford (1964).

[15] Laffargue D., Bourée F., Chevalier B., Roisnel T. and Bordère S., JAC 271-273, 444 (1998).

[16] Landau L. D. and Lifschitz E. M., «Statistical Physics», Chapter 3, Pergamon Press, Oxford (1959).

[17] Boccara N., «Symétries brisées : théorie des transitions avec paramètre d'ordre », Hermann, Paris (1976).

[18] Desclaux J. P. and Freeman A. J., JMMM 8, 119 (1978).

[19] Bordère S., Bourée F., Chevalier B. and Etourneau J., JMMM 253, 15 (2002).

[20] El Khayati N., Cherkaoui El Moursli R., Rodríguez-Carvajal J., André G., Blanchard N., Bourée F., Collin G. and Roisnel T., Eur. Phys. J. B 22, 429 (2001).

[21] El Khayati N., Rodríguez-Carvajal J., Bourée F., Roisnel T., Cherkaoui R., Boutfessi A. and Boukhari A., Solid State Sciences 4, 1273 (2002).

[22] Rousse G., Rodríguez-Carvajal J., Wurm C. and Masquelier C., Chem. Mater. 13, 4527 (2001). 
[23] Rousse G., Rodríguez-Carvajal J., Wurm C. and Masquelier C., Solid State Sci. 4, 973 (2002).

[24] Blake G. R., Chapon L. C., Radaelli P. G., Park S., Hur N., Cheong S.-W. and Rodríguez-Carvajal J., Phys. Rev. B 71, 214402 (2005).

[25] Sivardière J., «Description de la symétrie, des groupes de symétrie aux structures fractales », EDP Sciences, 2-86883-721-2 (2004).

[26] Sivardière J., «Symétrie et propriétés physiques, du principe de Curie aux brisures de symétrie », EDP Sciences, 2-86883-722-0 (2004).

[27] Bertaut E. F., Acta Cryst A 24, 217 (1968).

[28] Sikora W., Bialas F. and Pytlik L., J. Appl. Cryst. 37, 1015 (2004).

[29] Wills A., Physica B 276-278 (2000).

[30] Rodríguez-Carvajal J., Physica B 192, 55 (1993).

[31] Rodríguez-Carvajal J., Materials Science Forum 378-381, 268 (2001).

[32] Mirambet F., Gravereau P., Chevalier B., Trut L. and Etourneau J., JAC 191, L1 (1993).

[33] Rossat-Mignod J., «Magnetic Structures», in Methods of Experimental Physics, Neutron Scattering, Volume 3 (Academic Press, New York, 1987).

[34] Schweizer J., J. Phys. IV France 11, Pr9-105 (2001).

[35] Wills A., J. Phys. IV France 11, Pr9-133 (2001). 